\section{Class I histone deacetylases (HDAC1-3) are histone lysine delactylases}

\author{
Carlos Moreno-Yruela ${ }^{1}+$, Di Zhang ${ }^{2}+\neq$, Wei Wei $^{3}$, Michael Bæk ${ }^{1}$, Wenchao Liu ${ }^{2}$, Jinjun Gao ${ }^{2}$, \\ Daniela Danková ', Alexander L. Nielsen ${ }^{1} \S$, Julie E. Bolding ${ }^{1}$, Lu Yang ${ }^{2}$, Samuel T. Jameson ${ }^{1}$, \\ Jiemin Wong ${ }^{3}$, Christian A. Olsen ${ }^{1 *}$, Yingming Zhao ${ }^{2 *}$
}

\begin{abstract}
Lysine L-lactylation [K(L-la)] is a newly discovered histone mark stimulated under conditions of high glycolysis, such as the Warburg effect. $\mathrm{K}(\mathrm{L}-\mathrm{la})$ is associated with functions that are different from the widely studied histone acetylation. While K(L-la) can be introduced by the acetyltransferase p300, histone delactylases enzymes remained unknown. Here, we report the systematic evaluation of zinc- and nicotinamide adenine dinucleotide-dependent histone deacetylases (HDACs) for their ability to cleave $\boldsymbol{\varepsilon}-\mathrm{N}$-L-lactyllysine marks. Our screens identified HDAC1-3 and SIRT1-3 as delactylases in vitro. HDAC1-3 show robust activity toward not only K(L-la) but also K(D-la) and diverse short-chain acyl modifications. We further confirmed the de-L-lactylase activity of HDACs 1 and 3 in cells. Together, these data suggest that histone lactylation is installed and removed by regulatory enzymes as opposed to spontaneous chemical reactivity. Our results therefore represent an important step toward full characterization of this pathway's regulatory elements.
\end{abstract}

Copyright @ 2022

The Authors, some rights reserved; exclusive licensee American Association for the Advancement of Science. No claim to original U.S. Government Works. Distributed under a Creative Commons Attribution NonCommercial License 4.0 (CC BY-NC)

\section{INTRODUCTION}

Emerging lines of evidence suggest that metabolic end products and intermediates can have signaling functions in addition to their cognate roles. A metabolite can exert its function covalently through intrinsic chemical reactivity $(1,2)$ or enzyme-catalyzed reactions (3). Classic examples of the latter include acetyl-coenzyme A (CoA) and $S$-adenosylmethionine (SAM), which can be used by acetyltransferases for lysine acetylation and by methyltransferases for lysine methylation, respectively $(3,4)$. Other metabolites, such as nicotinamide adenine dinucleotide $\left(\mathrm{NAD}^{+}\right)$and $\alpha$-ketoglutarate, can serve as cofactors and regulate the activities of corresponding deacetylases and demethylases (5). L-Lactate, traditionally known as a metabolic waste product, has recently been found to play important roles in metabolism. L-Lactate production regenerates the NAD ${ }^{+}$ consumed by glycolysis within cells. The shuttling of L-lactate between different organs and cells serves as a major circulating carbohydrate source that plays important roles in normal physiology and in cancer (6-8). L-Lactate is massively induced under hypoxia and during the Warburg effect $(7,9,10)$, which are associated with many cellular processes and are closely linked to diverse diseases including neoplasia, sepsis, and autoimmune diseases (11). Nevertheless, the nonmetabolic functions of L-lactate in physiology and disease, especially during Warburg effect, remain largely unknown.

\footnotetext{
Center for Biopharmaceuticals and Department of Drug Design and Pharmacology, Faculty of Health and Medical Sciences, University of Copenhagen, Universitetsparken 2, DK-2100 Copenhagen, Denmark. 'Ben May Department for Cancer Research, The University of Chicago, Chicago, IL 60637, USA. ${ }^{3}$ Shanghai Key Laboratory of Regulatory Biology, Institute of Biomedical Sciences and School of Life Sciences, East China Normal University, Shanghai 200241, China.

*Corresponding author. Email: yingming.zhao@uchicago.edu (Y.Z.); cao@sund. ku.dk (C.A.O.)

†These authors contributed equally to this work.

¥Present address: State Key Laboratory of Protein and Plant Gene Research, School of Life Sciences and Peking-Tsinghua Center for Life Sciences, Peking University, Beijing 100871, China.

§Present address: Institute of Chemical Sciences and Engineering, Ecole Polytechnique Fédérale de Lausanne (EPFL), Lausanne CH-1015, Switzerland.
}

We recently reported that L-lactate is a precursor that can label and stimulate histone lysine $\varepsilon-N$-L-lactylation $[\mathrm{K}(\mathrm{L}-\mathrm{la})](12)$. Data suggest that L-lactate is transformed into L-lactyl-CoA (13) and transferred onto histones by acetyltransferases such as p300 (Fig. 1A) (12). Therefore, like acetyl-CoA and histone lysine acetylation (Kac) (14), histone K(L-la) represents another example showing that acyl-CoA species can affect gene expression directly via histone posttranslational modification (PTM). In addition, histone K(L-la) has different kinetics from those of histone (Kac) during glycolysis (12). Histone $\mathrm{K}(\mathrm{L}-\mathrm{la})$ is induced by hypoxia and the Warburg effect, serving as a feedback mechanism to promote homeostatic gene expression in the late phase of macrophage polarization (12). Our data therefore indicate that histone $\mathrm{K}(\mathrm{L}-\mathrm{la})$ is a physiologically relevant histone mark and has unique biological functions.

In addition to L-lactate [with $(S)$ configuration], its structural isomer D-lactate [with $(R)$ configuration] is also found in cells, although at much lower concentration (11 to $70 \mathrm{nM}$ concentration, compared to typical millimolar concentration of L-lactate, which can reach up to $40 \mathrm{mM}$ in some cancer cells) $(15,16)$. D-Lactate is formed primarily from methylglyoxal (MGO) through the glyoxalase pathway $(17,18)$ and is overproduced in rare conditions, including certain cases of short bowel syndrome. Under these unusual circumstances, D-lactate can reach $3 \mathrm{mM}$ concentration or higher in plasma $(16,19) . S$-D- $(R)$-Lactylglutathione, an intermediate in the glyoxalase pathway, can react nonenzymatically to install K(D-la) PTMs on glycolytic enzymes (Fig. 1A) (20). Nevertheless, five lines of evidence suggest that histones are L-lactylated rather than D-lactylated: (i) the huge difference in cellular concentration of the precursor metabolites, L-lactate versus D-lactate; (ii) the specificity of the antibodies used in our work toward K(L-la) ( 100-fold or higher; Fig. 1B); (iii) the fact that histone lactylation can be stimulated and labeled by L-lactate (12); (iv) the specific genomic localization of histone $\mathrm{K}(\mathrm{L}-\mathrm{la})$ that excludes the possibility of random, spontaneous chemical installation (12); and (v) the fact that K(D-la) is enriched solely in cytosolic proteins that are in close contact with $S$-D-lactylglutathione (20), which is not the case for histones. Despite the progress, key regulatory mechanisms of histone K(L-la) 
A

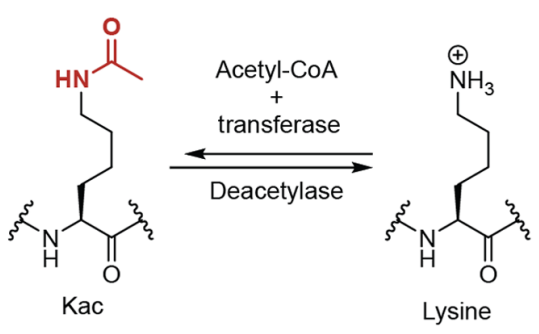

D

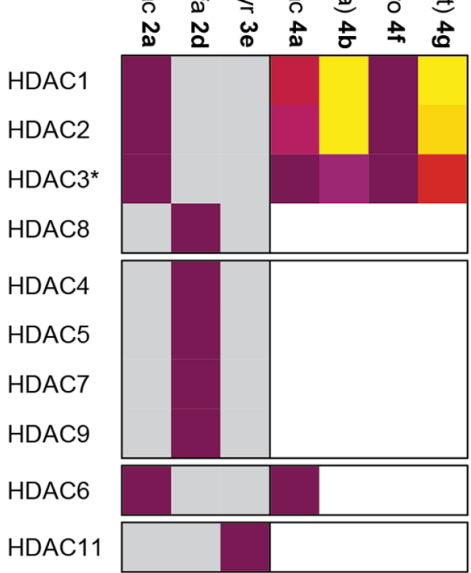<smiles>CCCC(CCCCNC(=O)[C@H](C)O)NC(C)(C)C</smiles>

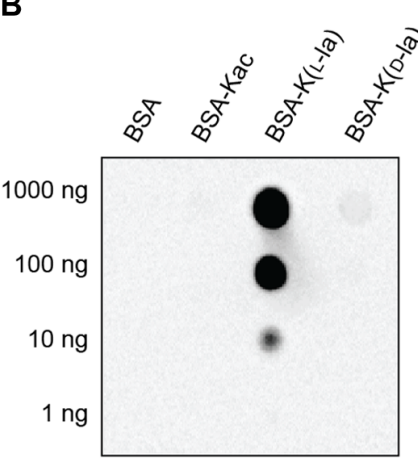

C<smiles>CCCCC(CC)NC(C)C</smiles>

Pan Kla

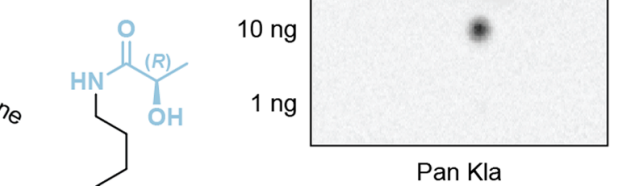

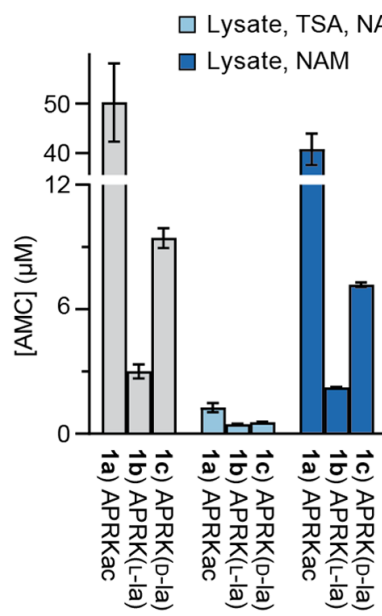
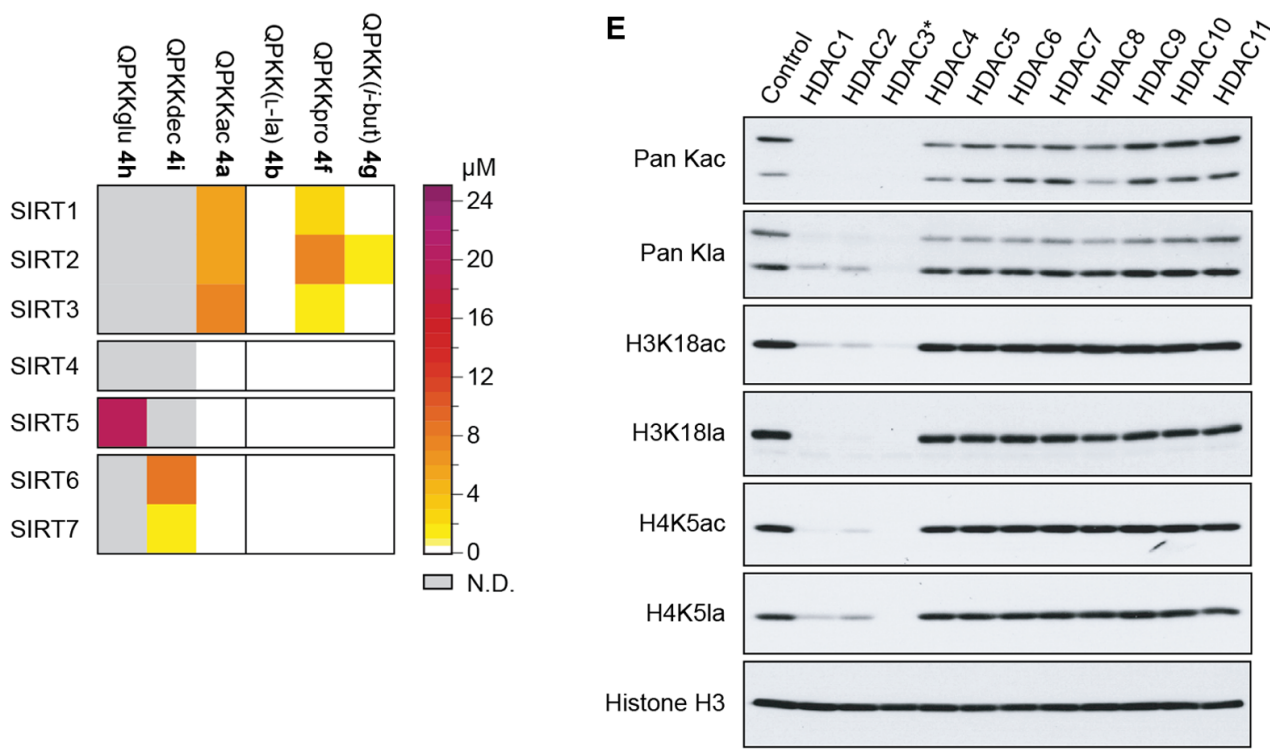

Fig. 1. In vitro screening for delactylase activity. (A) Reversible posttranslational modification of lysine to $\varepsilon-N$-acetyllysine (Kac), $\varepsilon-N$-L-lactyllysine [K(L-la)], and E-N-D-lactyllysine [K(D-la)]. (B) Dot blot assay using the pan anti-Kla antibody and bovine serum albumin (BSA) that were unmodified or modified with Kac, K(L-la), or K(D-la) PTMs. (C) Conversion of 7-amino-4-methylcoumarin (AMC)-conjugated Kac (1a), K(L-la) (1 b), and K(D-la) (1c) substrates (see fig. S2 for structures) by HEK293T whole-cell lysates and inhibition with an HDAC inhibitor [trichostatin A (TSA)] or a pan-sirtuin inhibitor (NAM). Data represent means \pm SD $(n=4)$. (D) Deacylase activity screening using short AMC-conjugated peptides, including positive controls for each recombinant enzyme (left part of heat maps), a K(L-la) substrate (4b), and substrates bearing short aliphatic modifications (4f and $\mathbf{4 g}$ ). Heatmaps represent mean values of AMC concentration ( $n=2)$ (see fig. S2 for substrate structures and fig. S3 for bar graphs). N.D.: not determined. (E) Deacylase activity screening using core histones from HeLa cells and antibodies against Kac and K(L-la) modifications, with histone $\mathrm{H} 3$ as loading control (4-hour reaction; see fig. S1 A for data after 1-hour incubation). *HDAC3 incubated with the deacetylase activation domain (DAD) of NCoR2.

remain unknown, including the enzymes that remove this modification in the cell (Fig. 1A).

Mammals express two families of lysine deacylases with 18 enzymes in total. Histone deacetylases (HDAC1-11, grouped into classes I, II, and IV) are $\mathrm{Zn}^{2+}$ dependent (21), while the sirtuins (SIRT1-7, class III HDACs) are dependent on $\mathrm{NAD}^{+}$as cosubstrate (22). Among these, several isozymes exhibit preferential enzymatic activities against non-Kac acylations. For example, SIRT5 is an efficient lysine demalonylase, desuccinylase, and deglutarylase but not deacetylase (23-26), and HDAC8, HDAC11, SIRT2, and SIRT6 harbor substantial activities against long-chain acyl modifications (27-30). HDAC1-3, in addition to their efficient deacetylase activity, also remove $\varepsilon$ - $N$-crotonyllysine (Kcr) and $\varepsilon-N$-D- $\beta$-hydroxybutyryllysine [K(D-bhb)] PTMs (31-34). Therefore, it is likely that enzymes from the lysine deacylase classes may be able to catalyze the removal of histone lactyl modifications.

Here, we report a screening of all 18 HDACs for potential delactylase activities using fluorophore-coupled peptides, fluorophore-free histone peptides, and extracted histones as substrates. We show that class I HDAC1-3 are the most efficient lysine delactylases in vitro and that $\mathrm{HDAC} 1$ and $\mathrm{HDAC} 3$ have site-specific delactylase activity in cells. These findings support that histone $\mathrm{K}(\mathrm{L}-\mathrm{la})$ modification is a regulatory and dynamic epigenetic mechanism. HDAC1-3 harbor activities in vitro toward diverse acyllysine groups including 
aliphatic and hydroxylated modifications of two to five carbons. In addition, even though we detect minor delactylase activity by SIRT1-3 in vitro, the pan-sirtuin inhibitor nicotinamide (NAM) does not affect the delactylase activity of a cell lysate or the histone $\mathrm{K}(\mathrm{L}-\mathrm{la})$ levels in cells. Our data suggest that HDAC1-3 are the main delactylases in the cell.

\section{RESULTS \\ Delactylase activities of $\mathrm{Zn}^{2+}$ - and $\mathrm{NAD}^{+}$-dependent human HDACs \\ Delactylase activities of cell lysates}

Lactylation of the side chains of lysine residues has been found on histones and other proteins $(12,20)$, but no efficient hydrolytic enzyme has been identified for this modification so far. To address this question, we first examined whether enzymes in the lysate of a human cell line [human embryonic kidney (HEK) 293T] were able to hydrolyze $\varepsilon-N$-lactyllysine modifications from short fluorophorecoupled peptide sequences derived from histone 3 [H3 ${ }_{15-18}$ : Ac-APRK(L-la)-AMC (7-amino-4-methylcoumarin) (1b) and Ac-APRK(D-la)-AMC (1c)]. The whole-cell lysate was indeed able to remove the lactyl modifications, albeit to a lower extent than the corresponding Kac modification (1a) (Fig. 1C). Substrate 1c with $\mathrm{K}(\mathrm{D}$-la) modification was converted to a larger extent than its K(L-la) counterpart, suggesting the presence of more efficient hydrolases of $\mathrm{K}(\mathrm{D}-\mathrm{la})$ in the cell. Treatment with the broad-spectrum HDAC inhibitor trichostatin A (TSA) (35) or the pan-sirtuin inhibitor NAM (36) indicated that the $\mathrm{Zn}^{2+}$-dependent HDACs are more likely to be responsible for these activities, because inhibition of sirtuin activity with NAM did not substantially change the outcome of the deacylation events (Fig. 1C).

\section{Delactylase activities of recombinant zinc-dependent HDACs} and sirtuins toward peptide substrates

Encouraged by these initial results, we then performed a screen of the activities of recombinant HDAC enzymes against known fluorogenic control substrates for each isozyme $(29,35,37-39)$ together with a set of fluorophore-conjugated p53 $317-320$ peptides (Ac-QPKKAMC) featuring small acyl-lysine modifications (Fig. 1D), because this sequence has been shown to be appropriate for both zincdependent HDACs and sirtuins $(29,37)$. Class I HDAC1-3 and sirtuins SIRT1-3 cleaved the aliphatic $\varepsilon-N$-propionyllysine (Kpro, $4 \mathbf{f}$ ) and, to some extent, $\varepsilon$ - $N$-isobutyryllysine $[\mathrm{K}(i$-but $), \mathbf{4 g}]$ modifications $(29,40)$. However, only HDAC1-3 exhibited measurable activity against the $\mathrm{K}(\mathrm{L}-\mathrm{la})$ substrate $(\mathbf{4 b})$ in these assays.

\section{Delactylase activities of recombinant zinc-dependent HDACs} and sirtuins toward histone substrates

To corroborate the results, we carried out in vitro delactylation assays using 18 recombinant HDACs and core histones as substrates, followed by Western blot analysis. We first evaluated specificities of anti-L-lactyllysine antibodies using bovine serum albumin (BSA) preparations that were either unmodified, acetylated, L-lactylated, or D-lactylated. We showed that the anti-L-lactyllysine antibody has more than 100-fold higher affinity for K(L-la)-modified than K(D-la)modified BSA and $~ 1000$-fold selectivity versus Kac-modified BSA (Fig. 1B). Thus, here, unless specified, all the Kla and lactylation will suggest the $\mathrm{L}$-isomer instead of the $\mathrm{D}$-isomer or their mixture.

Our enzymatic results indicated that HDAC1-3 substantially decrease the overall Kac and Kla levels on histones and also drastically reduce the levels of $\mathrm{H} 3 \mathrm{~K} 18$ and $\mathrm{H} 4 \mathrm{~K} 5$ L-lactylation (Fig. 1E and fig. S1A). In addition, we detected robust L-delactylation by SIRT1-3 to overall histone lactylation as well as H3K18(L-la) and H4K5(L-la) (fig. S1, B and C).

Together, our preliminary data showed that HDAC1-3 and, to some extent SIRT1-3, have robust in vitro delactylase activity and that $\mathrm{HDAC} 1-3$ are the most efficient enzymes among the 18 human recombinant enzymes in vitro. Thus, $\mathrm{HDAC} 1-3$ were selected for further investigation for their delactylase activity in vitro and in cells.

\section{HDAC3 is the most efficient eraser of both L- and D-lactyl-lysine in vitro}

To take a further look at the delactylase activity of HDAC1-3, we decided to investigate their delactylase activities compared to deacetylation. To this end, we synthesized and tested additional fluorogenic, histone-based substrates $\left(\mathrm{H}_{10-12}\right.$ : Ac-LGK-AMC; $\mathrm{H3}_{6-9}$ : Ac-TARK-AMC) with the Kac, K(L-la), and K(D-la) modifications, respectively (please consult the Supplementary Methods for synthetic details). The activity of HDAC1/2 caused limited conversion of the lactylated substrates compared to the acetylated counterparts and showed a slight preference for K(D-la) over $\mathrm{K}$ (L-la). HDAC3, on the other hand, converted L- and D-lactylated substrates to an extent more similar to that of acetylated substrates (Fig. 2A).

We then determined the delactylase efficiency of each isozyme. Conversion of several K(L-la) and K(D-la) substrates by HDAC3 had exceeded $30 \%$ in the previous experiment (initial substrate concentration: $50 \mu \mathrm{M}$ ), which indicates that the steady state was not maintained during the assay. Thus, to gain accurate kinetic insight, conversions of the L- and D-lactylated Ac-LGK-AMC substrates $\mathbf{2 b}$ and $2 \mathrm{c}$ were studied using an enzyme-coupled continuous assay (41). This revealed that $\mathrm{HDAC1}-3$ generally remove the $\mathrm{K}(\mathrm{D}-\mathrm{la})$ modification more efficiently than K(L-la) (Fig. 2B and fig. S4). In agreement with the end-point assay data, HDAC3 was the least sensitive to the configuration of the chiral center in the modification (3.5-fold higher $k_{\text {cat }} / K_{M}$ for $\mathbf{2 c}$ than for $\mathbf{2 b}$, compared to $>6$-fold for HDAC1/2) and was also the most efficient delactylase enzyme across all HDACs (Fig. 2C).

The delactylase and deacetylase activities of HDAC1-3 were also studied with longer, nonfluorogenic peptides to account for the potential effect of the fluorophore on enzyme activity and further characterize the site-specific delactylase activity of these enzymes. Six different peptides from histones $\mathrm{H} 2 \mathrm{~B}$ (histone $2 \mathrm{~B}$ ), $\mathrm{H} 3$, and $\mathrm{H} 4$ were prepared by solid-phase peptide synthesis and site-selectively modified with Kac, K(L-la), and K(D-la) modifications, respectively (Fig. 3A; see the Supplementary Methods for synthetic details). The deacetylase and delactylase activities of HDAC1-3, measured by high-performance liquid chromatography (HPLC) after 1 hour of incubation (Fig. 3B), followed similar patterns for all three modifications and correlated with those observed with fluorogenic substrates. However, larger differences in conversion were obtained depending on the sequence of the peptides. The sequence requirements of HDAC1/2 were different than those of HDAC3: HDAC1/2 showed lower activity at the H3K23 (8) and H4K12 (10) sites than HDAC3, relative to other peptides, while the H3K9 (6), H3K18 (7), and $\mathrm{H} 4 \mathrm{~K} 8$ (9) substrates were converted to a larger extent by all three enzymes (Fig. 3C). The $\mathrm{N}$-terminal peptide of $\mathrm{H} 2 \mathrm{~B}$ with modification at lysine $5(\mathrm{H} 2 \mathrm{BK} 5 ; 5)$ was poorly recognized by HDAC1-3. SIRT1-3, which have been shown to remove K(L-la) modifications 

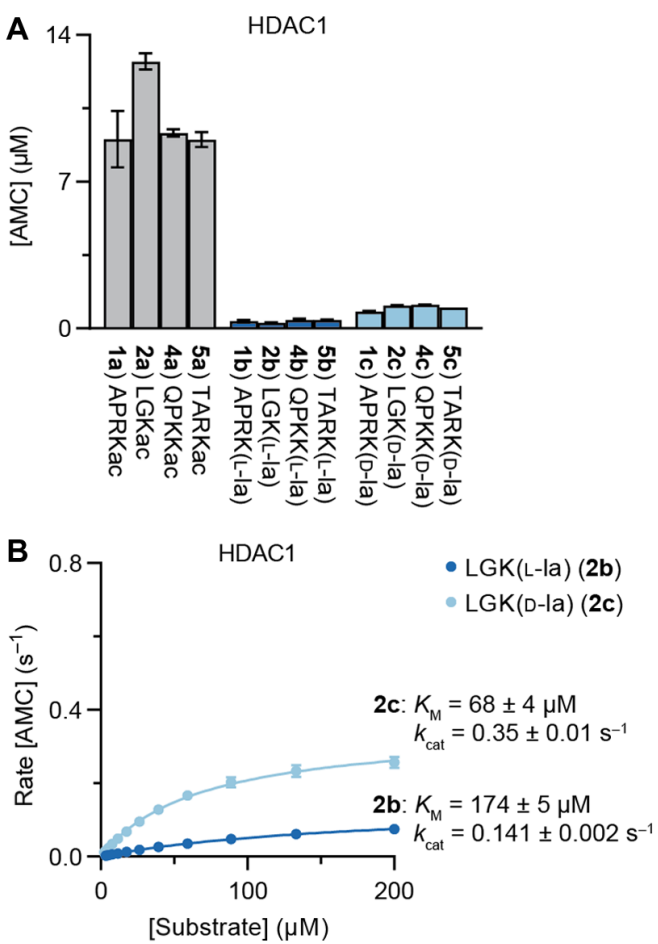
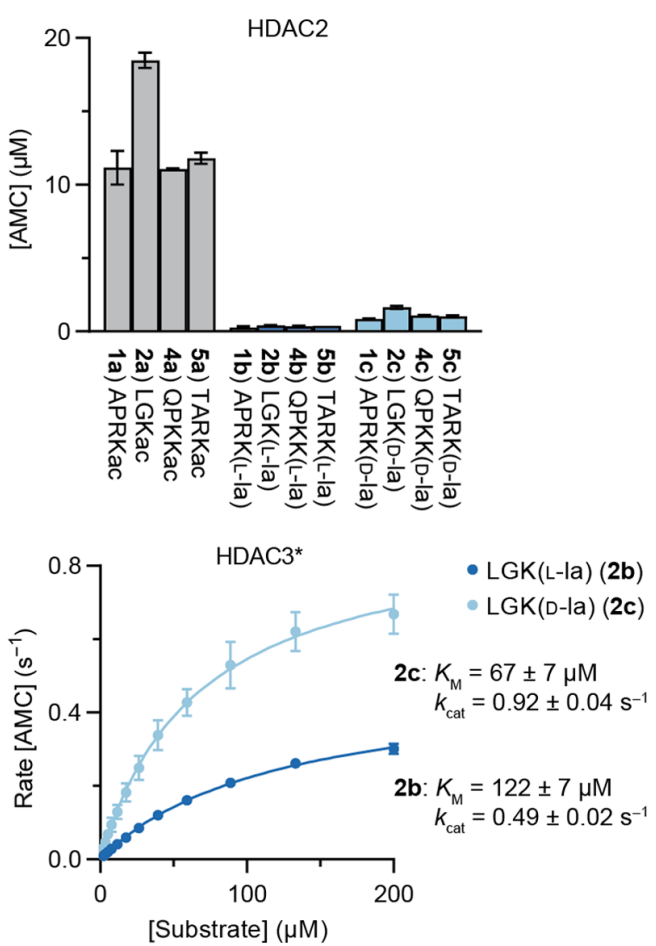

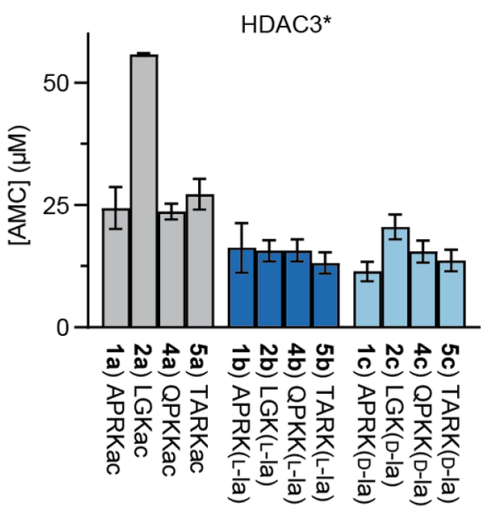

C

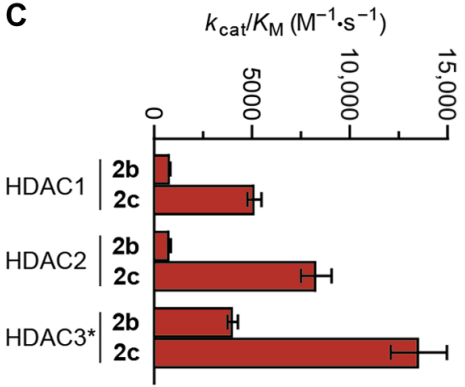

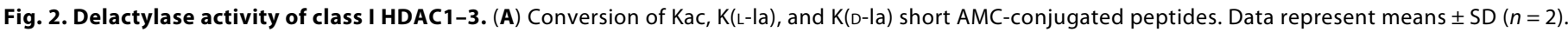

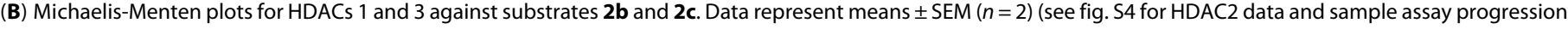

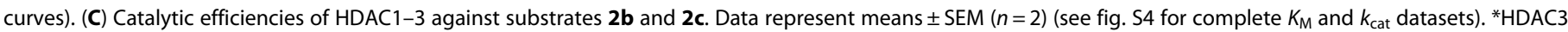
incubated with the DAD of NCoR2.

from a pyruvate kinase M2 (PKM2) peptide to some extent (42) and $\varepsilon-N$ - $\beta$-hydroxybutyryllysine (Kbhb) modifications from histone $\mathrm{H} 3$ peptides (43), only exhibited minor activity against the H3K18(L-la) (7b) and H3K18(D-la) (7c) peptides (Fig. 3D), in agreement with our initial screening data (Fig. 1D). Albeit, SIRT2 and, to a lesser extent, SIRT1 and SIRT3 were able to remove lactyl modifications from purified histones upon 4 hours of incubation (fig. S1). This could suggest that SIRT2 and SIRT3 serve as lysine delactylases in the cytosol and mitochondria (42), respectively, where HDAC1-3 are not present.

Conversion of $\mathrm{H} 3 \mathrm{~K} 9$ peptides $\mathbf{6} \mathbf{a}-\mathbf{c}$ was measured at several substrate concentrations after 10,15 , and $20 \mathrm{~min}$ to obtain steady-state conversion rates (Fig. 3E). Michaelis-Menten analysis again revealed a preference for $\mathrm{K}(\mathrm{D}-\mathrm{la})$ over $\mathrm{K}(\mathrm{L}-\mathrm{la})$ for $\mathrm{HDAC} 1 / 2$, although slightly less pronounced than that observed with fluorogenic substrates ( $<5$-fold higher $k_{\text {cat }} / K_{\mathrm{M}}$ for $\mathbf{6 c}$ than for $\mathbf{6 b}$ ) (Fig. $3 \mathrm{~F}$ ). In addition, HDAC3 was the most efficient delactylase when compared to its deacetylase activity, both at the $\mathrm{H} 3 \mathrm{~K} 9$ and $\mathrm{H} 4 \mathrm{~K} 12$ sites (Fig. 3F), which further supports our observations using short fluorogenic substrates. Furthermore, the efficiencies of HDAC3 for hydrolysis of K(L-la) and K(D-la) on nonfluorogenic peptides were $>1000$ fold higher than those reported for SIRT2 (42).

\section{HDAC3 removes various small aliphatic and hydroxylated acyl-lysine PTMs}

HDAC3, in association with the NCoR (nuclear receptor corepressor)/ SMRT (silencing mediator for retinoid and thyroid receptor) nuclear corepressor complex, is a potent lysine deacetylase (44), but it also harbors additional catalytic activities. Recent work has revealed that histone Kcr and Kbhb modifications are installed by p300 $(34,45)$. HDAC3 hydrolyzes Kcr and Kbhb in vitro and in human cells (31-34), and other aliphatic acyl modifications of up to 10 carbons in length can be removed as well (29). To elaborate upon this ability and compare its delactylase activity to those reported before, we prepared a collection of $\mathrm{H}_{10-12}$ substrates with small aliphatic and hydroxylated modifications to study the substrate scope of HDAC3 further (Fig. 4A) (29, 31, 46).

Michaelis-Menten experiments provided highly diverse steadystate parameters and highlighted critical structural substrate requirements (Fig. 4B). Short linear aliphatic PTMs (Kac, 2a; Kpro, 2f; Kbut, 2j; and Kcr, 2m) exhibited Michaelis-Menten constants $\left(K_{\mathrm{M}}\right)$ less than $10 \mu \mathrm{M}$, in agreement with previous studies for this isozyme (31). These four substrates, as well as the $\varepsilon$ - $N$-trifluoroacetyllysine modification (Ktfa, 2d) used for screening of HDAC inhibitors (35), were all cleaved with efficiencies $\left(k_{\text {cat }} / K_{\mathrm{M}}\right)$ over $100,000 \mathrm{M}^{-1} \cdot \mathrm{s}^{-1}$. The addition of a single hydroxy group to Kac to give $\varepsilon-N$-glycolyllysine (Kgc, 2n) markedly increased the substrate $K_{\mathrm{M}}$ value but still afforded high efficiency becuse of an unusually high catalytic rate constant $\left(k_{\text {cat }}=15.3 \pm 0.3 \mathrm{~s}^{-1}\right.$; Fig. 4B). Branched aliphatic chains [K( $i$-but $)$, $2 \mathbf{g}$; $\mathrm{K}(i$-val $), 2 \mathbf{k}$ ] provided lower $k_{\text {cat }}$ values, resulting in a $\sim 10$-fold lower efficiency (29). The efficiency was also lower against branched modifications containing hydroxy groups [K(L-la), 2b; K(L-bhb), 2 ; Khib, 2o; etc.], resulting from a combination of lower $k_{\text {cat }}$ and higher $K_{\mathrm{M}}$ values. Both $\mathrm{K}(\mathrm{D}$-bhb) and $\varepsilon-N$-2-hydroxyisobutyryllysine (Khib) have been found on histones and connect cell metabolism with gene expression regulation $(47,48)$. Kbhb is also cleaved by SIRT1-3, although with lower efficiency in vitro (43). SIRT3 exhibits slight preference for K(L-bhb) over K(D-bhb) (43), whereas HDAC3 


\begin{tabular}{|c|c|}
\hline 5) H2BK5X & $\mathrm{H}-\mathrm{PEPAXSAPA}-\mathrm{WW}-\mathrm{NH}_{2}$ \\
\hline 6) $\mathrm{H} 3 \mathrm{~K} 9 \mathrm{X}$ & AC-W-KQTARXSTGG-W-NH \\
\hline 7) $\mathrm{H} 3 \mathrm{~K} 18 \mathrm{X}$ & AC-W-GGKAPRX $Q L A T K-W-\mathrm{NH}_{2}$ \\
\hline 8) $H 3$ K $23 \mathrm{X}$ & Ac-W-KQLATXAARK-W-NH ${ }_{2}$ \\
\hline 9) $\mathrm{H} 4 \mathrm{~K} 8 \mathrm{X}$ & Ac-W-RGKGG $\mathbf{X} G L G K-W-\mathrm{NH}_{2}$ \\
\hline 10) $\mathrm{H} 4 \mathrm{~K} 12 \mathrm{X}$ & $\mathrm{AC}-\mathrm{W}-\mathrm{GKGLGX} \mathrm{GGAK}-\mathrm{W}-\mathrm{NH}_{2}$ \\
\hline
\end{tabular}
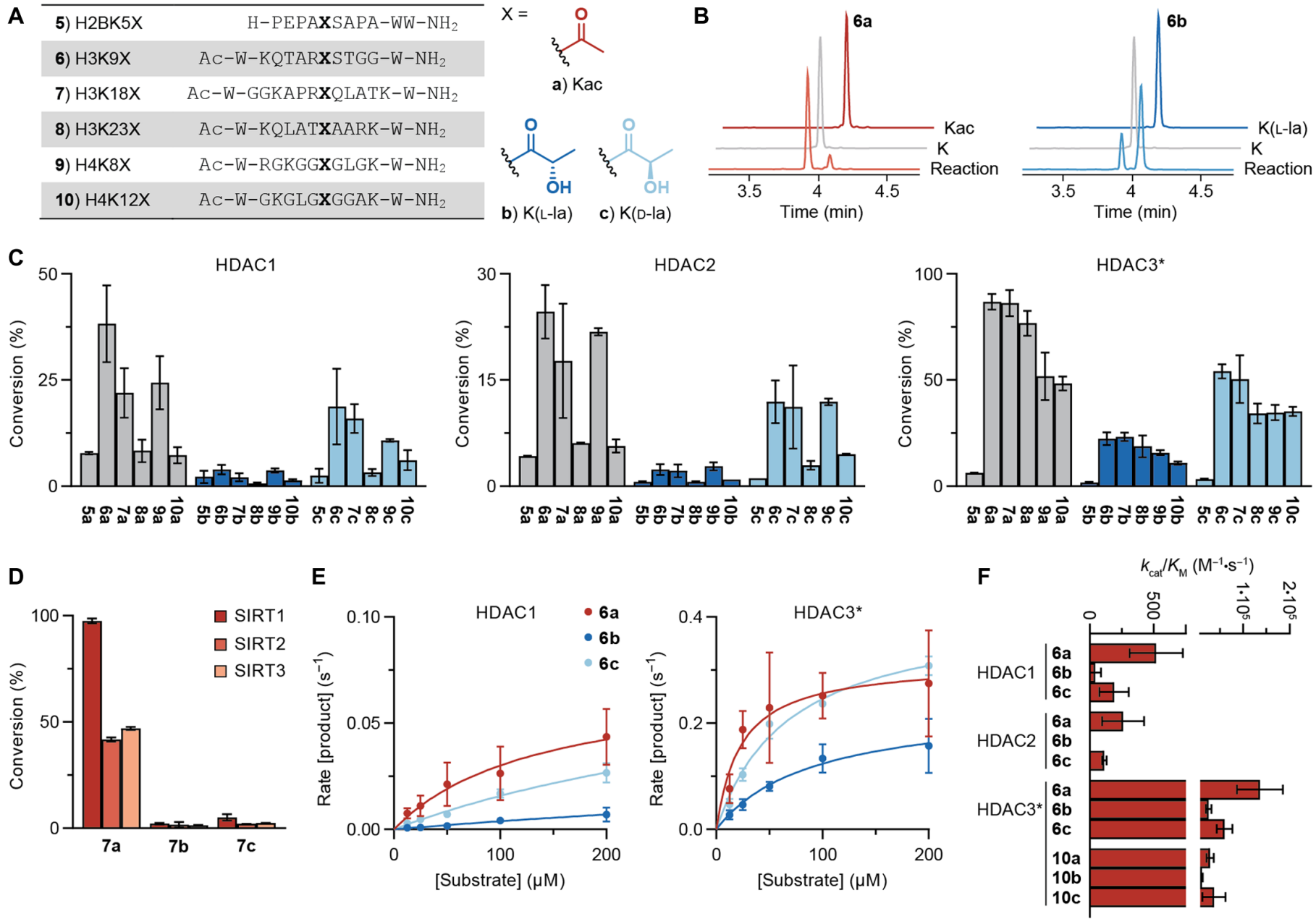

Fig. 3. Delactylase activities of HDAC1-3 at different histone sites. (A) Synthesized histone peptide sequences, with $X=K a c$ (a), K(L-la) (b), or K(D-la) (c). (B) Sample deacetylation and delactylation HPLC assay traces (60-min reaction of $50 \mathrm{nM} \mathrm{HDAC3/NCoR2}$ with $50 \mu \mathrm{M}$ peptide $\mathbf{6 a}$ or $\mathbf{6 b}$ ). (C) Relative conversion of Kac-, K(L-la)-, or K(D-la)-containing histone peptides. Data represent means \pm SD $(n=2)$. (D) Relative conversion of Kac-, K(L-la)-, or K(D-la)-containing H3K18 peptide by SIRT1-3. Data represent means \pm SD $(n=2)$. (E) Michaelis-Menten plots for HDAC1-3 against substrates $\mathbf{6 a}, \mathbf{6 b}$, and $\mathbf{6 c}$. Data represent means \pm SEM $(n=2)$ (see fig. S5 for HDAC2 data). (F) Catalytic efficiencies of HDAC1-3 against substrates $\mathbf{6 a - c}$ and 10a-c. Data represent means \pm SEM ( $n=2)$ (see fig. S5 for Michaelis-Menten plots of substrates $10 a-c$ and numerical data). ${ }^{*} \mathrm{HDAC} 3$ incubated with the DAD of NCoR2.

shows opposite selectivity (Fig. 4B), which could indicate complementary regulatory mechanisms by HDACs and sirtuins (34).

Overall, HDAC3 showed efficiencies less than $20,000 \mathrm{M}^{-1} \cdot \mathrm{s}^{-1}$ for all hydroxylated acyl-lysine PTMs except Kgc (Fig. 4B). These data categorized the L- and D-delactylase activities of HDAC3 among the less-efficient catalytic conversions for HDACs in vitro $(31,35)$ yet similar to those of HDAC8 or sirtuins recorded for their validated cellular targets in similar assay formats $(49,50)$.

To rationalize the preference of HDAC3 for K(D-la) over K(L-la), small-molecule substrate mimics containing both PTMs were docked into the crystal structure of HDAC3 bound to the deacetylase activation domain (DAD) of NCoR2 [Protein Data Bank (pdb) 4A69; Fig. 4C] (51). Both modifications were accommodated in the HDAC3 active site in a conformation similar to that of a Kac substrate bound to HDAC8 (52). The HDAC8 structure was chosen for comparison because it is the only class I HDAC isozyme cocrystallized with an acylated substrate thus far. The hydroxy group of the $\mathrm{K}$ (D-la) modification could be positioned to form a hydrogen bond with histidine 134 (H134) (Fig. 4C, pink molecule), which was not possible with the hydroxy group of $\mathrm{K}(\mathrm{L}-\mathrm{la})$ as it pointed in the opposite direction (Fig. 4C, green molecule). The H134 residue is conserved in $\mathrm{Zn}^{2+}$-dependent HDACs and involved in transition state stabilization (53). Thus, this stereoisomer-specific interaction could explain the higher hydrolytic efficiency of HDAC1-3 for K(D-la) over K(L-la).

\section{Quantification of HDAC3-mediated histone delactylation in vitro}

To corroborate the results in peptide-based in vitro assays, we examined the delactylase activity of HDAC 3 on histones by incubation of recombinant HDAC3 with acid-extracted histones from HeLa cells (54). We found that global histone lactylation levels were decreased by HDAC 3 , an activity that could be inhibited by both the broad-spectrum HDAC inhibitor TSA and the HDAC1-3-specific inhibitor apicidin (Fig. 5A) (35). To gain further insights into which lactylation sites are regulated by HDAC3, we used a stable isotope 


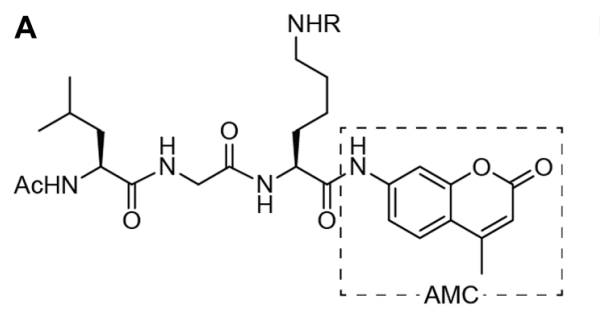

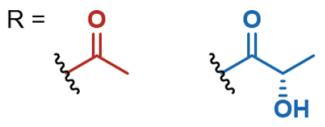<smiles>CCC(=O)C(C)O</smiles><smiles>[14CH3]C(=O)C(F)(F)F</smiles>

2a) Kac

2b) $\mathrm{K}(\mathrm{L}-\mathrm{la})$

2c) $K(\mathrm{D}-\mathrm{la})$

2d) Ktfa

LGKacyl, $\mathrm{H}_{10-12}$<smiles>CCC(C)=O</smiles><smiles>CCC(=O)C(C)C</smiles><smiles>CCCC(C)=O</smiles>

2f) Kpro

2g) $\mathrm{K}(i$-but $)$

2j) Kbut

2k) K(i-val)<smiles>CCC=O</smiles><smiles>C/C=C/C(C)=O</smiles><smiles>CCC(=O)CO</smiles><smiles>CCC(=O)C(C)(C)O</smiles><smiles>CCC(=O)CC(C)O</smiles><smiles>CCC(=O)C[C@@H](C)O</smiles>

21) Kfor

2m) Kcr

2n) Kgc

2o) Khib

2p) $\mathrm{K}(\mathrm{L}-\mathrm{bhb})$

2q) $K(D-b h b)$

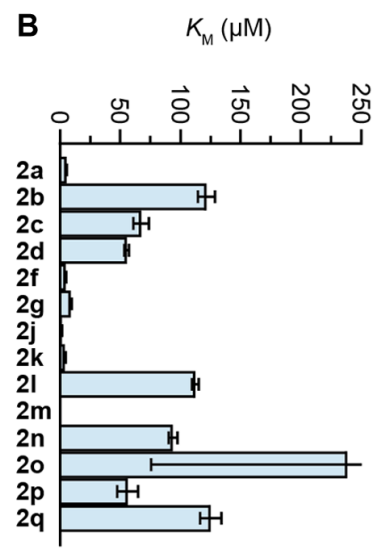

$k_{\text {cat }}\left(\mathrm{s}^{-1}\right)$

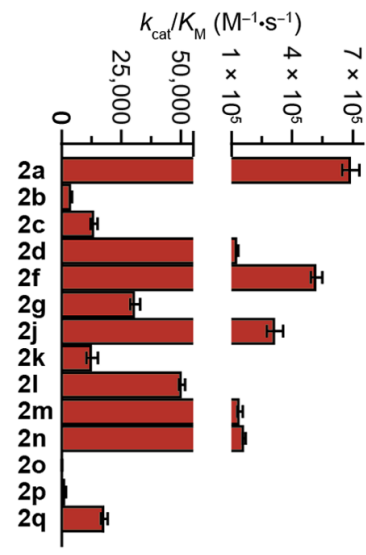

C

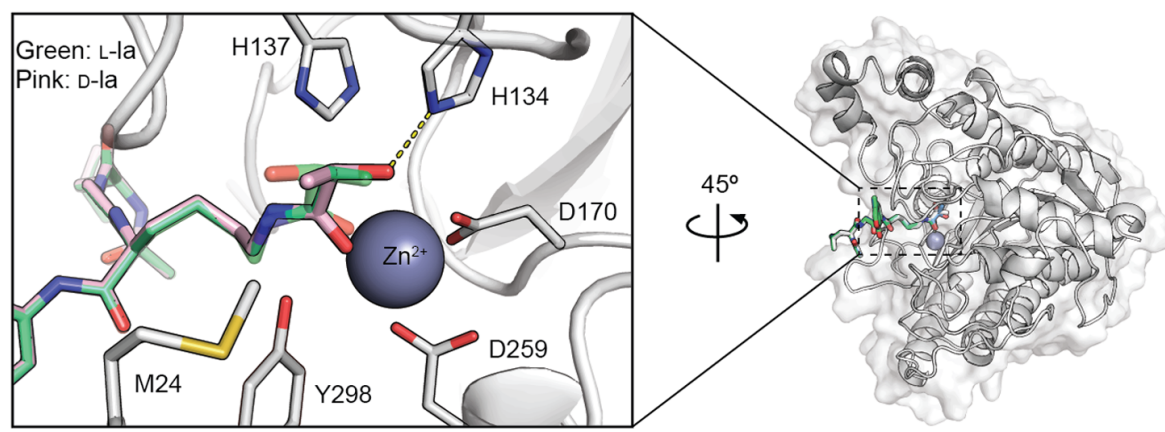

Fig. 4. Acyl-lysine substrate scope for HDAC3. (A) Structure of $\mathrm{H} 4_{10-12}$ (Ac-LGK-AMC) substrates. (B) Kinetic parameters of HDAC3/NCoR2 against $\mathrm{H} 4_{10-12}$ substrates with various acyl-lysine modifications. Data represent means \pm SEM $(n=2)$ (see fig. S4 for Michaelis-Menten curves and numerical data). (C) Docking of K(L-la) (green) and $\mathrm{K}(\mathrm{D}-\mathrm{la}$ ) (pink) substrate mimics into the crystal structure of HDAC3 (pdb 4A69). A hydrogen bond between $\mathrm{H} 134$ and the hydroxy group of the K(D-la) modification is predicted.

labeling by amino acids in cell culture (SILAC)-based quantification method (55). Equal numbers of histones extracted from "light"or "heavy"-labeled cells were incubated with or without recombinant HDAC3 for 4 hours. Then, after tryptic digestion, the light and heavy histone peptides were mixed at a ratio of $1: 1(\mathrm{~m} / \mathrm{m})$ and analyzed by HPLC-mass spectrometry (MS). To eliminate potential bias on PTM levels caused by SILAC labeling, we performed both forward and reverse labeling, and in both replicates, histone acetylation and lactylation were efficiently removed by HDAC3 (Fig. 5, B to D). We detected $39 \mathrm{~K}(\mathrm{~L}$-la) peptides on histones, among which $31 \mathrm{~K}(\mathrm{~L}$-la) peptides were decreased by more than $90 \%$ in response to HDAC 3 in at least one replicate, and $38 \mathrm{~K}(\mathrm{~L}-\mathrm{la})$ peptides showed $>50 \%$ decrease in both replicates (Fig. 5B and table S1). Lactylated Nterminal $\mathrm{H} 2 \mathrm{~B}$ peptides were decreased to a lesser extent than most $\mathrm{H} 3$ and $\mathrm{H} 4$ peptides (table S1), which was in agreement with the lower conversion of H2BK5 substrates (Fig. 3C). Overall, our results demonstrate that HDAC3 has robust histone delactylase activity in vitro.

\section{HDAC1 and HDAC 3 site-specifically remove $\varepsilon-\mathrm{N}$-lactyllysine modifications on histones in the cell}

We then explored whether the delactylase activity of HDAC1-3 is maintained in living cells in culture. Treatment of HeLa cells for 5 hours with broad-spectrum HDAC inhibitors (sodium butyrate 
A

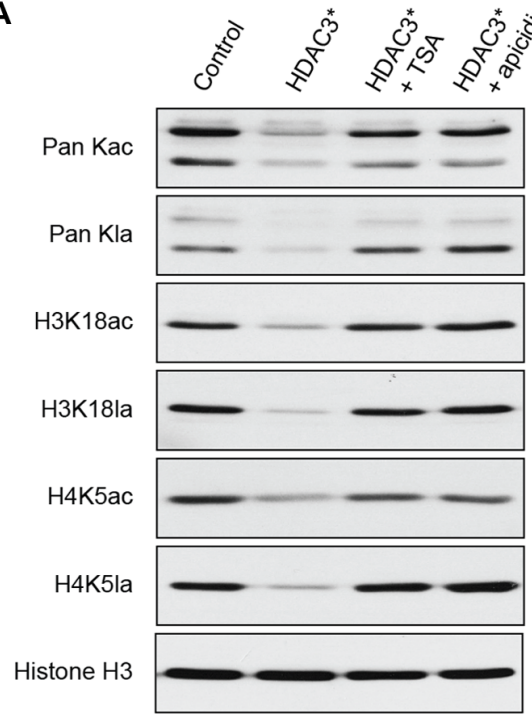

B

Forward labeling Light histone (L): control Heavy histone $(\mathrm{H})$ : HDAC3*

$\operatorname{HDAC}^{*}(\mathrm{H})$ vs. control $(\mathrm{L})$

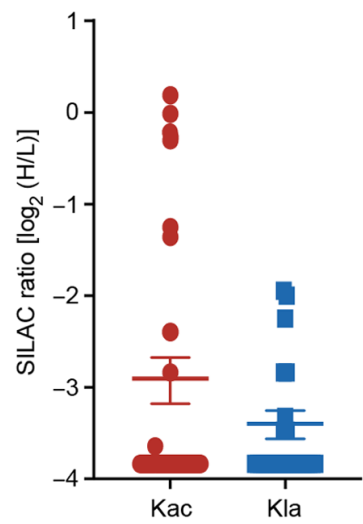

Reverse labeling

Light histone (L): HDAC3*

Heavy histone $(\mathrm{H})$ : control

Control (H) vs. HDAC3* (L)

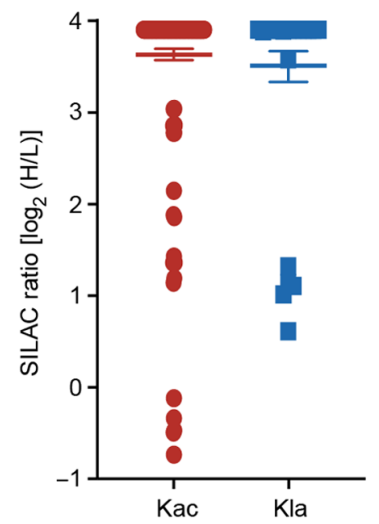

C

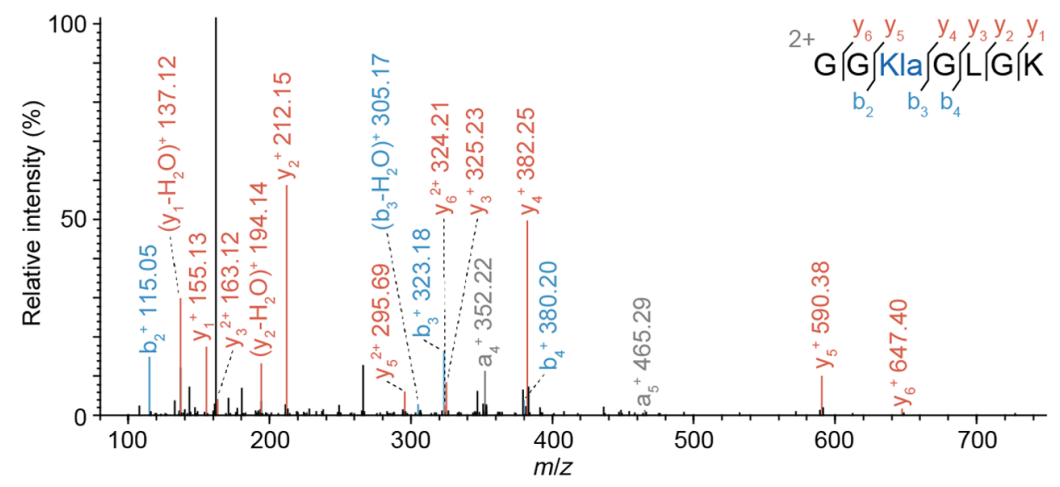

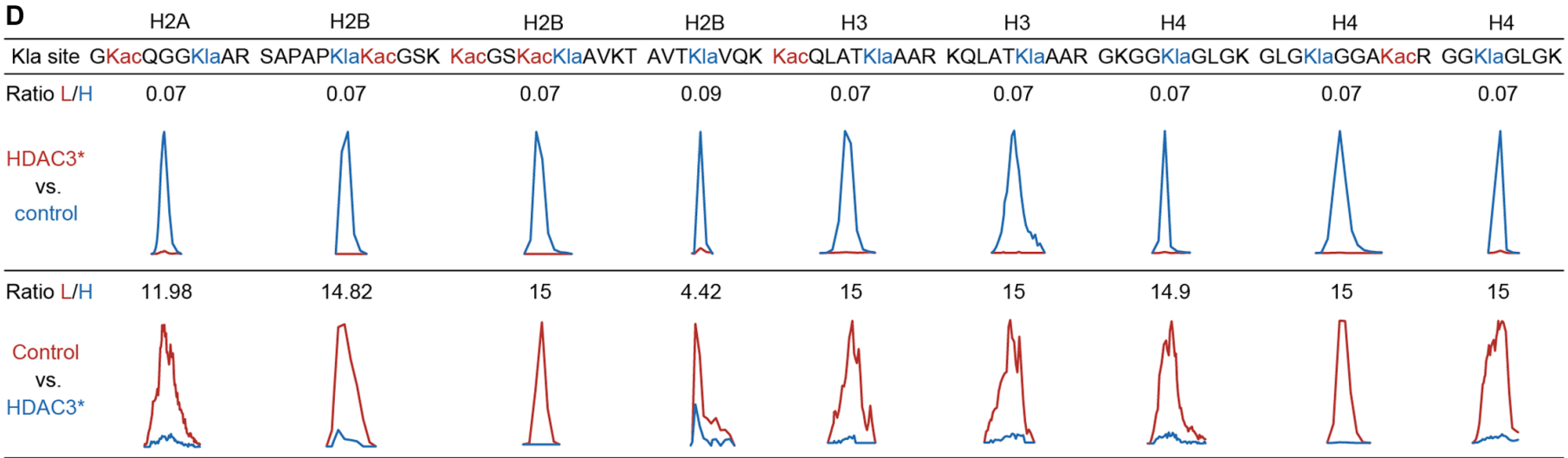

Fig. 5. Mass spectrometric identification and quantification of in vitro HDAC3-catalyzed delactylation targets on core histones. (A) Changes in lactylation and acetylation of histones (overall, and at the sites H3K18 and H4K5) upon HDAC3 treatment and inhibition with TSA and apicidin. (B) Forward and reverse MS quantification of Kla and Kac sites, with or without HDAC3. (C) A representative MS/MS spectrum of the histone H4 peptide GGKlaGLGK. (D) Representative MS quantification from in vitro histone deacylation products shown in (B). A full list of quantified peptides can be found in table $\mathrm{S} 1$.

and TSA) increased global histone lactylation levels, and the HDAC13-specific inhibitor apicidin also enhanced lactylation (Fig. 6A and fig. S6). In contrast, the class IIa HDAC inhibitor TMP195 and the sirtuin inhibitor NAM failed to affect histone lactylation levels substantially (Fig. 6A). While the lack of effect of TMP195 on Kac levels is expected, it is less obvious why the NAM sirtuin inhibitor does not affect histone Kac levels. However, this is in full agreement with previous findings from Choudhary and co-workers, investigating the impact of NAM $(10 \mathrm{mM})$ on histone Kac levels by quantitative MS (56). Nevertheless, the results taken together indicate that HDAC1-3 are lysine delactylases in cell nuclei.

To further dissect the action of each isozyme, HDAC1-3 were overexpressed in HeLa cells, as verified by Western blot (Fig. 6B). Overexpression of each isozyme resulted in a reduction in the overall levels of histone acetylation (pan Kac) and crotonylation (pan Kcr) $(32,57)$, but no change was observed for overall levels of histone 
A

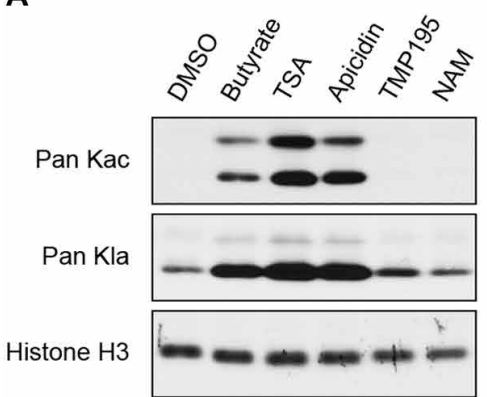

B

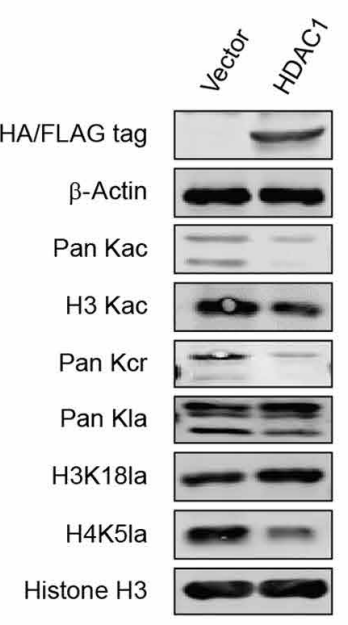

C

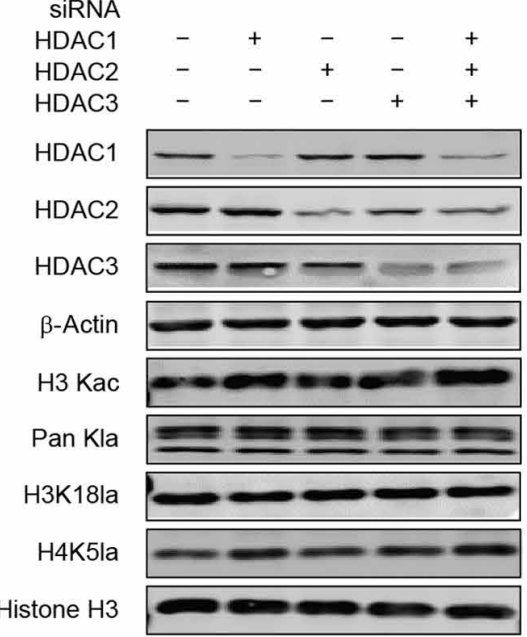

D

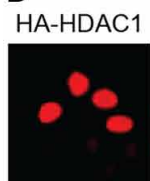

HA-HDAC1

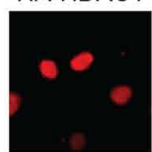

HA-HDAC1

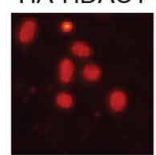

HA-HDAC1

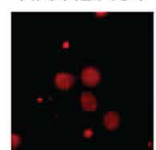

HA-HDAC1

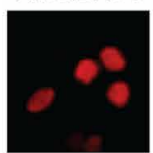

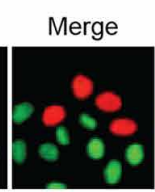

Pan $\mathrm{Kcr}$
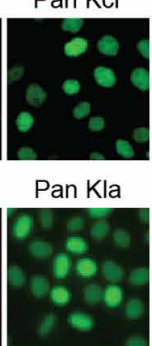

H3K18la

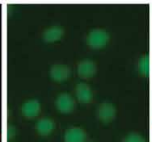

H4K5la

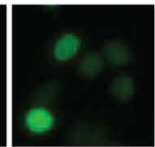

Merge

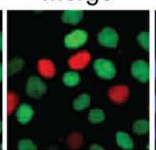

Merge

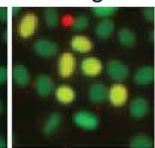

Merge

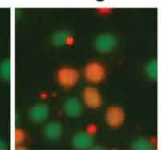

Merge

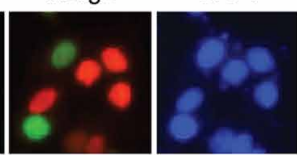

Scale bar, $50 \mu \mathrm{m}$

DAPI

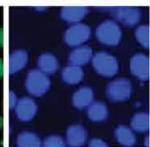

DAPI

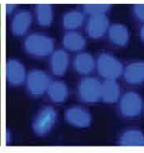

DAPI

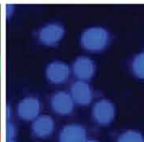

E
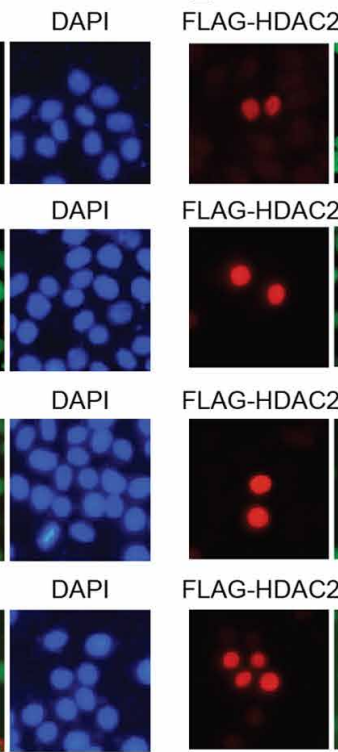

FLAG-HDAC2 H4K5la
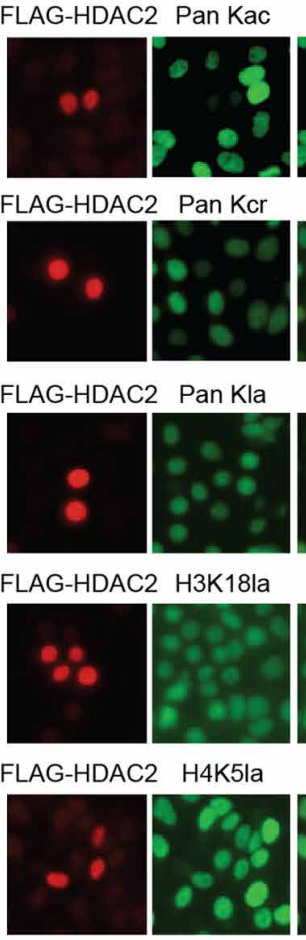

FLAG-HDAC2 Pan Kcr
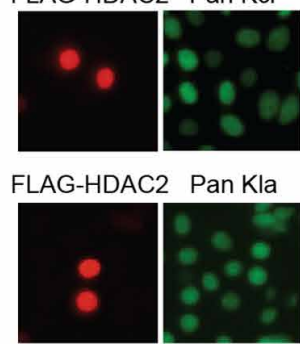

FLAG-HDAC2 H3K18la

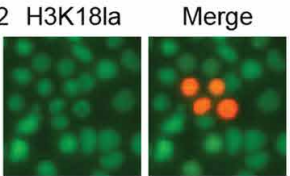

Merge

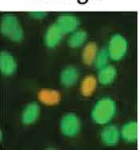

Scale bar, $50 \mu \mathrm{m}$
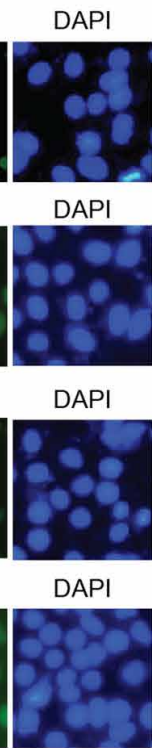

DAPI

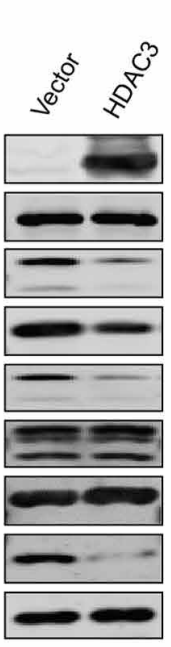

$\mathbf{F}$

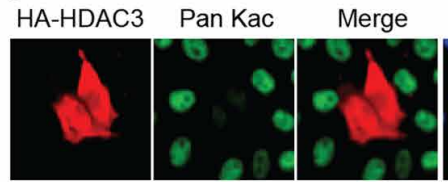

DAPI
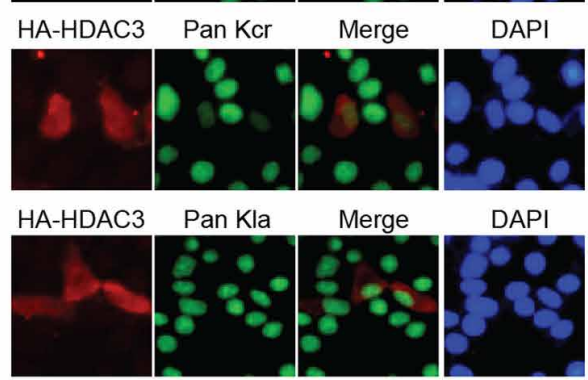

DAPI

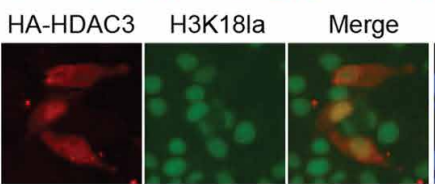

DAPI
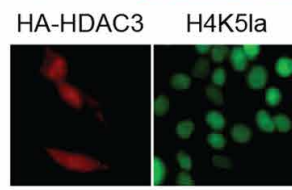

Merge

DAPI

Fig. 6. In cellulo deacetylase, decrotonylase, and delactylase activities of HDAC1-3. (A) Changes in overall lactylation and acetylation of histones in HeLa cells upon 5-hour treatment with pan-HDAC inhibitors (sodium butyrate, $2 \mathrm{mM} ; \mathrm{TSA}, 1 \mu \mathrm{M}$ ), the HDAC1-3-selective inhibitor apicidin (1 $\mu \mathrm{M}$ ), the class Ila-selective inhibitor TMP195 $(5 \mu \mathrm{M})$, or the pan-sirtuin inhibitor NAM $(10 \mathrm{mM}$ ) (see fig. $\mathrm{S} 6 \mathrm{for}$ full Western blots and blots with longer exposure time). (B) Western blot analysis of core histones from HeLa cells with or without transfection of HA-tagged HDAC1, FLAG-tagged HDAC2, or HA-tagged HDAC3. (C) Western blot analysis of core histones from HeLa cells with or without small interfering RNA (siRNA)-mediated knockdown of HDACs 1, 2, 3, or their combination (72-hour treatment). Top blots correspond to whole-cell lysates and show the level of ectopically expressed HDAC (B) or HDAC knockdown (C) relative to $\beta$-actin (see fig. S6 for full Western blots). (D to F) Immunostaining images of HeLa cells transfected with HA-tagged HDAC1, FLAG-tagged HDAC2, or HA-tagged HDAC3 and the levels of histone acetylation, crotonylation, and lactylation. DAPI: 4',6-diamidino-2-phenylindole (nuclear stain).

lactylation (pan Kla) (Fig. 6B). Site-specific lactylation levels at $\mathrm{H} 4 \mathrm{~K} 5$, on the other hand, were reduced by HDAC1-3, while the H3K18la modification remained unchanged in all three experiments (Fig. 6B; see fig. S7 for H4K5 antibody dot blot). These results were corroborated by immunofluorescence staining and analysis of individual nuclei (Fig. 6, D to F). HDAC1/2 constructs were expressed in the nuclei, while HDAC 3 was also observed in the cytosol, as reported $(58,59)$. In these experiments, cells with detectable levels of transfected HDACs 1 and 3 also showed strong reduction of Kac, Kcr, and H4K5la levels, but not for the H3K18la mark or global Kla modification. Thus, overexpression of a single class I HDAC is not sufficient for altering the overall levels of histone 
lactylation to an extent detectable by Western blot or immunofluorescence. Moreover, HDAC1-3 exhibit site-selective activities in the cell, which are different from those observed in vitro (Fig. 3C). A plausible explanation for this effect would be the formation of nuclear multiprotein complexes able to direct the delactylase activity toward specific histone sites (60).

To validate our findings from the overexpression experiments, we performed RNA interference experiments to knock down HDAC1-3. Western blot analysis revealed that knockdown of HDAC1 or HDAC3 but not HDAC2 slightly increased histone H4K5 lactylation levels, and triple knockdown of HDAC1-3 led to a stronger effect on $\mathrm{H} 4 \mathrm{~K} 5$ lactylation (Fig. 6C). We reasoned that the extended times of transfection (72-hour treatment) could enable compensation of the individual knockdowns by other HDACs having similar function and that this could explain the weak effects observed. Thus, we then used an HDAC3-selective proteolysis-targeting chimera (PROTAC) adapted from the literature (61) to perform a much faster knockdown (fig. S8). HDAC3 knockdown by PROTAC DD-I22 did not increase lactylation levels to a substantial extent, as judged by Western blot (fig. S9), but it led to a slight increase in multiple $\mathrm{Kac}$ and Kla sites measured by SILAC-MS after 4 hours of treatment (Fig. 7). These data suggest that HDAC3 regulates histone lactylation in cells not only at $\mathrm{H} 4 \mathrm{~K} 5$ but also at multiple sites. In sum, our assays suggest that HDAC1 and HDAC3 have delactylase activity in cells.

\section{DISCUSSION}

Metabolic intermediates are chemical sources for histone PTMs and affect gene expression $(1,14)$. In particular, the growing landscape of histone acyl modifications has revealed a dynamic and diverse network of metabolic modifications that regulate transcription in health and disease $(12,47,48)$. The metabolic importance of L-lactate and its association with disease have been known for almost a century, but it was only recently discovered as a precursor
A

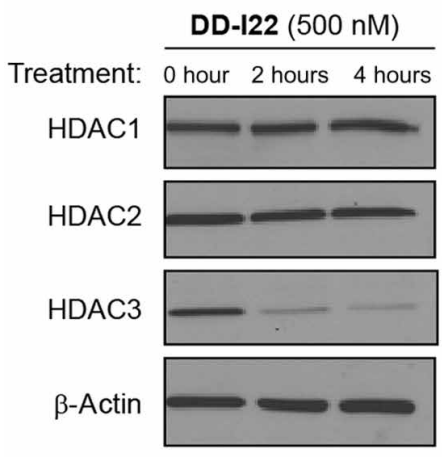

B Light histone (L): DMSO Heavy histone $(H)$ : DD-I22 Treatment: 4 hours

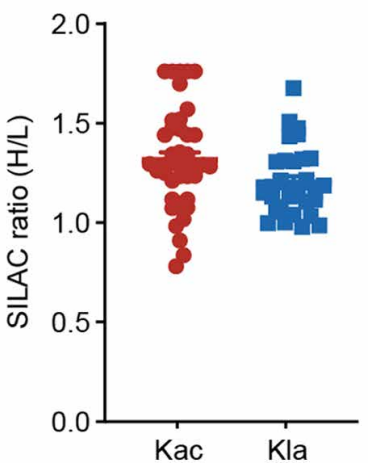

Fig. 7. Selective degradation of HDAC3 and mass spectrometric quantification of in vivo delactylation targets on core histones. (A) Changes in HDAC1-3 concentration upon treatment of HEK293 cells with PROTAC DD-122 at $500 \mathrm{nM}$ for the indicated time (see fig. 58 for chemical structure and optimization of degradation conditions in HeLa and HEK293T cells). (B) MS quantification of Kac and Kla sites on core histones extracted from HEK293 cells upon treatment with DD-I22 (500 nM) for 4 hours (see fig. S10 for quantification after 2 hours). for stimulating histone K(L-la) marks that, in turn, modulate gene expression (12). K(L-la) modifications, like other histone acylations, can be installed by histone acetyltransferases (HATs) $(12,34,45)$, but little is known about their reversibility and turnover. Identification of key regulatory enzymes, lactyltransferases, and delactylases, as well as their substrate proteins, will be a stepping stone for dissecting the functions of L-lactate signaling pathways in the future.

Here, we report the first systematic in vitro screening of delactylase activity using synthetic peptides and core histones as substrates. The nuclear HDAC1-3 were the most robust and efficient delactylases, while the only activity detected for the sirtuins in vitro was on purified histones. These results were corroborated in cells by the effect of HDAC and sirtuin inhibitors with different specificities. Nevertheless, it remains to be addressed in the future whether sirtuins 1 to 3 can act as delactylases at protein sites complementary to HDACs or in other cellular compartments, as it has been proposed for SIRT2 in the cytosol (42). By overexpression and knockdown experiments, we found that HDACs 1 and 3 exhibit delactylase activity in the cell, but our experiments also showed that individual isozymes could not alter the overall levels of histone lactylation to a large extent. The reason for a seemingly preferential regulation of H4K5la by HDACs 1 and 3 in cells remains unclear, although recent evidence has suggested that H4K5 residues are often modified by various acylations and are finely tuned by cellular metabolism (62). Furthermore, when using targeted degradation of HDAC3 with a selective PROTAC, broader Kla up-regulation was observed by SILAC-MS, albeit to a small extent. Together, these data suggest the existence of site-specific K(L-la) regulatory pathways, and potentially, other unknown delactylases exist in cells.

HDAC1-3 perform their catalytic functions as part of large multicomponent protein complexes in the nucleus (60). These complexes consist of scaffolding proteins that allow the HDACs to interact with other epigenetic enzymes, such as demethylases and transcription factors (60). The activity of the HDACs is enhanced when engaged in their cognate complexes (44), and standard recombinant preparations of HDAC3 include the directly interacting DAD domain of NCoR2 for increased activity. This might explain the enhanced delactylase activity observed in vitro for HDAC3 compared to $\mathrm{HDAC} 1 / 2$. It has also been shown that HDAC1-3 display distinct Kac substrate selectivity upon interaction with different complex partners (63), which could be the reason for the discrepancies observed between in vitro and cellular data. HDACs 1 and 3, but not HDAC2, can prominently change the levels of lactylation at the $\mathrm{H} 4 \mathrm{~K} 5$ site. $\mathrm{HDAC} 1 / 2$ are frequently present in the same multiprotein complex in the nucleus and are thought to compensate for each other in the regulation of histone $\mathrm{Kac}(60)$. Our data show that HDAC1/2 may have unique individual deacylation substrates and thus indicate distinct functions of these two enzymes in cells. However, it remains to be studied to what extent the formation of complexes directs HDAC activity toward specific histone K(L-la) sites.

$n$-Butyrate and other short-chain fatty acids modify gene transcription patterns via inhibition of HDACs, leading to histone hyperacetylation $(64,65)$. This epigenetic mechanism has also been attributed to L- and D-lactate (66) and to $\beta$-hydroxybutyrate (67). However, the concentrations necessary for achieving HDAC inhibition by these hydroxylated species are in the high millimolar range ( 100 -fold higher than that of $n$-butyrate) and often lead to minor changes in acetylation $(66,68,69)$. Alternatively, dynamic covalent 
modification of histones by L-lactyl-CoA and other activated acyl groups may explain their regulatory effects at physiological concentration.

L-Lactate governs lactate metabolism and signaling in humans (70), whose concentration (usually in the millimolar range) is typically more than 1000 times higher than D-lactate in cells (typically in the nanomolar range). L-Lactate is a known precursor for protein lysine L-lactylation (most likely through lactyl-CoA) (12), while MGO, but not D-lactate, is a precursor for lysine D-lactylation [via $S$-D-(R)-lactylglutathione] (20). L-Lactate labels proteins through enzyme-catalyzed reaction (likely via lactyl-CoA) $(12,13)$, which was demonstrated in nuclei but can most likely happen in the cytosol similar to other lysine acylation events, e.g., Kac (71). HDAC1-3 show remarkable activities toward diverse $\varepsilon$ - $N$-acyllysine groups including $\mathrm{K}(\mathrm{D}-\mathrm{la})$, and it remains to be confirmed whether these activities are physiologically relevant. Here, we demonstrate that HDAC1-3 are responsible for the reversible and dynamic regulation of histone L-lactylation. This result and the specificity of genomic localization of histone L-lactylation observed by chromatin immunoprecipitation sequencing (12) argue loudly that histone L-lactylation is likely the major pathway in nuclear lactylation. Together, these findings not only provide insight into the new activities of class I HDACs but also validate the regulation of histone K(L-la) by pharmacologically targetable enzymes, which lays the foundation for future functional studies of this modification and its associated regulatory pathways.

\section{MATERIALS AND METHODS Chemical synthesis}

Please find the synthesis and characterization of peptide substrates in the Supplementary Methods (72).

\section{Assay materials}

Fluorescence-based deacylase activity assays were performed in black low-binding 96-well microtiter plates (Corning half-area wells, Fisher Scientific, cat. \#3686), with duplicate series in each assay and each assay performed at least twice. Control wells without enzyme were included in each plate. HPLC-based deacylase activity assays were performed in microcentrifuge plastic tubes $(1.5 \mathrm{ml}$, Sarstedt AG, cat. \#72.690.001) and filtered through 0.45- $\mu \mathrm{m}$ PTFE (polytetrafluoroethylene) filters before HPLC injection (MachereyNagel GmbH \& Co., cat. \#729015), with each assay performed at least twice. Experiments were performed in Hepes buffer [50 mM Hepes/Na, $100 \mathrm{mM} \mathrm{KCl,} \mathrm{0.001 \%} \mathrm{(v/v)} \mathrm{Tween} \mathrm{20,} \mathrm{and} 0.2 \mathrm{mM}$ tris(2-carboxyethyl)phosphine (pH 7.4)] (35) or tris buffer [50 mM tris/Cl, $137 \mathrm{mM} \mathrm{NaCl}, 2.7 \mathrm{mM} \mathrm{KCl}$, and $1 \mathrm{mM} \mathrm{MgCl}_{2}(\mathrm{pH} \mathrm{8)}$ ] (Fluor de Lys buffer, Enzo Life Sciences) with BSA (Sigma-Aldrich, cat. \#A7030) as indicated for each assay. $\beta$-Nicotinamide adenine dinucleotide hydrate $\left(\mathrm{NAD}^{+}\right)$for sirtuin assays was from a commercial source (Sigma-Aldrich, cat. \#N7004), as well as trypsin (Sigma-Aldrich, cat. \#T1426). The following recombinant enzymes were acquired from BPS Bioscience (San Diego, CA): HDAC1 (full length, C-terminal His tag, cat. \#50051), HDAC2 (full length, C-terminal His tag, cat. \#50002), HDAC3/NCoR2 [full length, C-terminal His tag, with DAD domain of NCor2, N-terminal glutathione $S$-transferase (GST) tag, cat. \#50003], HDAC4 [amino acids (aa) 627 to 1084, N-terminal GST tag, cat. \#50004], HDAC5 (aa 656 to 1122, C-terminal His tag, cat. \#50005), HDAC6 (full length, C-terminal FLAG tag, cat. \#50056), HDAC7 (used only with extracted histones; aa 518-end, N-terminal GST tag, cat. \#50007), HDAC8 (full length, C-terminal His tag, cat. \#50008), HDAC9 (aa 604 to 1066, C-terminal His tag, cat. \#50009), HDAC10 (used only with extracted histones; aa 2 to 631, N-terminal FLAG tag, cat. \#50060), HDAC11 (full length, untagged, cat. \#50021), SIRT1 (aa 193 to 741, N-terminal GST tag, cat. \#50012), SIRT2 (aa 50 to 356, C-terminal His tag, cat. \#50013), SIRT3 (aa 102 to 399, N-terminal GST tag, cat. \#50014), SIRT4 (aa 25 to $314, \mathrm{~N}$-terminal GST tag, cat. \#50015), SIRT5 (used only with extracted histones; full length, N-terminal GST tag, cat. \#50016), SIRT6 (full length, N-terminal GST tag, cat. \#50017), and SIRT7 (used only with extracted histones; full length, C-terminal FLAG tag, cat. \#50018); or from Millipore (Temecula, CA): HDAC7 (used only with fluorogenic substrates; aa 383-end, N-terminal His tag, cat. \#14-832). Recombinant SIRT5 and SIRT7 used with fluorogenic substrates were produced and purified as described below. PTM antibodies anti-Kla (cat. \#PTM-1401), anti-Kac (cat. \#PTM-101), anti-Kcr (cat. \#PTM-501), and sequence-specific anti-H3K18la (cat. \#PTM-1406) and anti-H4K5la (cat. \#PTM-1407) were purchased from PTM Biolabs Inc. (Chicago, IL). Anti-histone H3 (cat. \#4499) and anti-actin (cat. \#4970) were purchased from Cell Signaling Technology Inc. (Danvers, MA); anti-FLAG (cat. \#F7425/F1804) was purchased from MilliporeSigma (Burlington, MA); antihemagglutinin (HA) (cat. \#SC-805) was purchased from Santa Cruz Biotechnology Inc. (Dallas, TX); and anti-HDAC1 (cat. \#A0238), anti-HDAC2 (cat. \#A2084), and anti-HDAC3 (cat. \#A2139) were purchased from ABclonal Inc. (Woburn, MA). Inhibitors were of commercial source: apicidin (Sigma-Aldrich, cat. \#A8851), entinostat (MS-275; TargetMol, cat. \#T6233), NAM (MilliporeSigma, cat. \#N0636), sodium butyrate (MilliporeSigma, cat. \#B5887), TMP195 (Cayman Chemical Company, cat. \#23242), and TSA (Tokyo Chemical Industry, cat. \#T2477, or MilliporeSigma, cat. \#T8552). Stocks were prepared in dimethyl sulfoxide (DMSO) (1 to $40 \mathrm{mM}$ ), and concentrations of peptide substrates were determined on the basis of absorbance $\left[\varepsilon_{280}(\operatorname{Trp})=5690 \mathrm{M}^{-1} \cdot \mathrm{cm}^{-1}\right.$ or $\varepsilon_{326}($ Ac-Lys-AMC $)=17783 \mathrm{M}^{-1} \cdot \mathrm{cm}^{-1}$ ] (73) using a Thermo Fisher Scientific NanoDrop instrument. Assay concentration of substrates and inhibitors were obtained by dilution from DMSO stock solutions in buffer, and appropriate concentration of enzyme was obtained by dilution of the stock provided by the supplier. Data analysis was performed using GraphPad Prism 8 or 9 .

\section{Expression and purification of SIRT5 and SIRT7}

pET100/D-TOPO vectors encoding Escherichia coli codon-optimized SIRT5 (aa 34 to 310 ) or SIRT7 (aa 1 to 400) and an N-terminal cleavage site for tobacco etch virus (TEV) protease were designed in GeneArt and purchased from Thermo Fisher Scientific. Proteins were expressed in E. coli BL21(DE3), and cells were harvested and disrupted by sonication for $6 \times 1 \mathrm{~min}$ in lysis buffer [SIRT5: $12.5 \mathrm{mM}$ $\mathrm{Na}_{2} \mathrm{HPO}_{4}, 1.8 \mathrm{mM} \mathrm{KH}_{2} \mathrm{PO}_{4}, 2.7 \mathrm{mM} \mathrm{KCl}, 137 \mathrm{mM} \mathrm{NaCl}, 1 \%$ Tween 20, and $15 \mathrm{mM} \beta$-mercaptoethanol ( $\mathrm{pH} \mathrm{7.6);} \mathrm{SIRT7:} 50 \mathrm{mM}$ tris, $400 \mathrm{mM} \mathrm{NaCl}, 20 \mathrm{mM} \beta$-mercaptoethanol, and $10 \%$ glycerol $(\mathrm{pH} 8)]$. The supernatants were filtrated and subjected to affinity chromatography using a 5-ml HisTrap HP His-tag purification column (GE Healthcare, cat. \#GE29-0510-21) operated by a peristaltic pump (Cytiva, cat. \#18111091) at $4^{\circ} \mathrm{C}$. Supernatants were run onto the column followed by $25 \mathrm{ml}$ of lysis buffer. SIRT 5 was eluted with a gradient of 0 to $50 \%$ elution buffer [SIRT5 lysis buffer with $500 \mathrm{mM}$ imidazole ( $\mathrm{pH} 7.6$ )], and SIRT7 was eluted with $25 \mathrm{ml}$ of 
elution buffer [SIRT7 lysis buffer with $500 \mathrm{mM}$ imidazole (pH 8)]. Fractions were collected using an ÄKTApurifier system with ÄKTA Frac-950 fraction collector (GE Healthcare Life Sciences). The eluted fractions were analyzed by gel electrophoresis and Coomassie blue staining, collected, dialyzed against lysis buffer using Spectra/Por 4 dialysis tubing [12- to $14-\mathrm{kDa}$ MWCO (molecular weight cutoff); Spectrum, cat. \#132697], and treated with TEV protease $(0.2 \mathrm{mg} / \mathrm{ml})$ overnight at $4^{\circ} \mathrm{C}$. Samples were then subjected to reverse affinity chromatography using a 5-ml HisTrap HP His-tag purification column at $4^{\circ} \mathrm{C}$. Flow-through and following washes were collected and concentrated to $<1 \mathrm{ml}$ using an Ultra-15 Centrifugal Filter unit with a cutoff value of $30 \mathrm{kDa}$ (Amicon, \#UFC903024). The purified SIRT7 was then stored at $-80^{\circ} \mathrm{C}$. The SIRT5 sample was subjected to gel filtration on a Superdex $75 \mathrm{HR}$ 10/30 column (Amersham) in buffer [25 mM tris/ $\mathrm{HCl}, 100 \mathrm{mM} \mathrm{NaCl}, 15 \mathrm{mM} \beta$-mercaptoethanol E, and $10 \%$ (v/v) glycerol (pH 7.5)] using the ÄKTApurifier system, and fractions containing SIRT5 were pooled, concentrated, and stored at $-80^{\circ} \mathrm{C}$. These enzymes were used only with fluorogenic substrates.

\section{Fluorogenic lysate deacylase assay}

HEK293T cells [American Type Culture Collection (ATCC)] were cultured under a humidified 5\% $\mathrm{CO}_{2}$ atmosphere in Dulbecco's modified Eagle's medium (DMEM; Thermo Fisher Scientific, cat. \#11965118) supplemented with 10\% (v/v) fetal bovine serum (FBS), $1 \%$ penicillin, and $1 \%$ streptomycin. For lysate preparation, media was aspirated, and cells were washed with phosphate-buffered saline (PBS) and treated with lysis buffer (1\% Triton X-100, 0.2\% SDS, and cOmplete EDTA-free protease inhibitor cocktail, COEDTAF-RO Sigma-Aldrich, in PBS). Upon scraping, lysates were collected in microcentrifuge tubes, sonicated with a Bandelin Sonopuls mini20 ( $2 \mathrm{~s}$ on, $2 \mathrm{~s}$ off, $80 \%$ amplitude, $1 \mathrm{~min}$ ), and centrifuged $(14,000 \mathrm{~g}$, $10 \mathrm{~min}, 4^{\circ} \mathrm{C}$ ), and protein concentrations of the supernatants were determined by the bicinchoninic acid assay (BCA1; Sigma-Aldrich). Lysates $(30 \mu \mathrm{g}$ per well) were then incubated with substrates $\mathbf{1 a}-\mathbf{c}$ $(50 \mu \mathrm{M})$ and $\mathrm{NAD}^{+}(500 \mu \mathrm{M})$ and TSA $(100 \mu \mathrm{M})$ or NAM $(10 \mathrm{mM})$ in tris buffer with BSA $(0.5 \mathrm{mg} / \mathrm{ml})$ at a final volume of $25 \mu \mathrm{l}$ per well. The reaction was incubated at $37^{\circ} \mathrm{C}$ for $60 \mathrm{~min}$, and then a solution of trypsin $(25 \mu \mathrm{l}, 5.0 \mathrm{mg} / \mathrm{ml}$; final concentration of $2.5 \mathrm{mg} / \mathrm{ml})$ and NAM ( $4 \mathrm{mM}$; final concentration of $2 \mathrm{mM}$ ) was added, and the assay development was allowed to proceed for $90 \mathrm{~min}$ at room temperature before fluorescence analysis. The data were analyzed to afford $[\mathrm{AMC}]$ relative to control wells without enzyme.

\section{Fluorogenic substrate screening assays}

The initial screening for substrate deacylation activity (Fig. 1D) was performed in tris buffer with BSA $(0.5 \mathrm{mg} / \mathrm{ml})$, with end-point AMC fluorophore release by trypsin. For a final volume of $25 \mu \mathrm{l}$ per well, acyl substrates $(50 \mu \mathrm{M})$ and $\mathrm{NAD}^{+}(500 \mu \mathrm{M}$; only for sirtuin assays) were added to each well, followed by a solution of the appropriate KDAC enzyme (HDACs: $50 \mathrm{nM}$; SIRTs 1, 2, and 5: $200 \mathrm{nM}$; SIRTs 3, 4, 6, and 7: $500 \mathrm{nM}$ ). The reaction was incubated at $37^{\circ} \mathrm{C}$ for $60 \mathrm{~min}$ (HDACs) or $120 \mathrm{~min}$ (sirtuins), then a solution of trypsin $(25 \mu \mathrm{l} ; 5.0 \mathrm{mg} / \mathrm{ml}$; final concentration of $2.5 \mathrm{mg} / \mathrm{ml}$ ) was added, and the assay development was allowed to proceed for $90 \mathrm{~min}$ at room temperature before fluorescence analysis. The data were analyzed to afford [AMC] relative to control wells without enzyme.

Follow-up screening with Kac, K(L-la), and K(D-la) fluorogenic substrates (Fig. 2A) was performed in Hepes buffer with BSA
$(0.5 \mathrm{mg} / \mathrm{ml})$, with end-point AMC fluorophore release by trypsin. Substrate $(50 \mu \mathrm{M})$ and enzyme $(2$ and $10 \mathrm{nM})$ were incubated at $37^{\circ} \mathrm{C}$ for $60 \mathrm{~min}$ in a final volume of $25 \mu \mathrm{l}$. Then, a solution of trypsin $(25 \mu \mathrm{l} ; 5.0 \mathrm{mg} / \mathrm{ml}$; final concentration of $2.5 \mathrm{mg} / \mathrm{ml})$ was added, and the assay development was allowed to proceed for $90 \mathrm{~min}$ at room temperature before fluorescence analysis. The data were analyzed to afford [AMC] relative to control wells without enzyme.

\section{Fluorogenic Michaelis-Menten assays}

Rate experiments for determination of kinetic parameters with AMC-containing substrates were performed in Hepes buffer with BSA $(0.5 \mathrm{mg} / \mathrm{ml})$. Trypsin concentration was optimized for HDAC1-3 at $100 \mu \mathrm{M}$ substrate concentration to ensure that trypsin-mediated $\mathrm{AMC}$ release was not the rate-limiting step while minimizing its effect on HDAC stability (41). For a final volume of $50 \mu \mathrm{l}$, substrate (2a, 2f: 100 to $1.74 \mu \mathrm{M} ; \mathbf{2 b}, \mathbf{2 c}: 200$ to $2.31 \mu \mathrm{M}$; 2d, 2g, 2j, 2k, 2l, 2n, 2o, 2p, 2q: 200 to $3.47 \mu \mathrm{M} ; 2 \mathrm{~m}$ : 25 to $0.43 \mu \mathrm{M} ; 1.5$-fold dilutions), trypsin $(10 \mu \mathrm{g} / \mathrm{ml})$, and enzyme (HDAC1: $30 \mathrm{nM}$; HDAC2: $15 \mathrm{nM}$; HDAC3 with 2a, 2d, 2f, 2n: 1 nM; with 2b, 2c, 2l: 5 nM; with 2g, 2j, 2k, 2q: $10 \mathrm{nM}$; and with $2 \mathrm{~m}, 2 \mathrm{o}, 2 \mathrm{p}$ : $20 \mathrm{nM}$ ) were added to a microtiter plate and immediately placed in the plate reader. In situ fluorophore release was monitored by fluorescence readings every $30 \mathrm{~s}$ for $60 \mathrm{~min}$ at $25^{\circ} \mathrm{C}$. Then, initial conversion rates $\left(v_{0}\right)$ were determined for each concentration, and data were fitted to the Michaelis-Menten equation to afford $K_{\mathrm{M}}$ and $k_{\text {cat }}$ values (41).

\section{HPLC-based substrate assays}

Substrate assays with nonfluorogenic peptide substrates were performed in Hepes buffer (HDACs) or tris buffer (sirtuins) without BSA, with end-point chromatographic determination of relative conversion. Substrate $(50 \mu \mathrm{M})$ and enzyme (HDAC1 and HDAC2: $250 \mathrm{nM}$; HDAC3: $50 \mathrm{nM}$; SIRT1: $50 \mathrm{nM}$; SIRT2 and SIRT3: $100 \mathrm{nM}$ ) were incubated at $37^{\circ} \mathrm{C}$ for $60 \mathrm{~min}$ in a final volume of 30 or $40 \mu \mathrm{l}$ in microcentrifuge tubes. Then, $\mathrm{MeOH} / \mathrm{HCOOH}[94: 6(\mathrm{v} / \mathrm{v})]$ was added to quench the reaction ( 15 or $20 \mu \mathrm{l}$; for 45 or $60 \mu \mathrm{l}$ of total volume), and samples were agitated on an orbital shaker and centrifuged at $20,000 \mathrm{~g}$ for $10 \mathrm{~min}$. Samples were then taken up with a plastic syringe, filtered, and analyzed by analytical HPLC on an Agilent 1260 Infinity II series system equipped with a diode array detector. A gradient with eluent III $\left[\mathrm{H}_{2} \mathrm{O} / \mathrm{MeCN} /\right.$ trifluoroacetic acid (TFA), 95:5:0.1, v/v] and eluent IV (0.1\% TFA in MeCN) rising linearly 0 to $40 \%$ during $t=1$ to 5 min was applied at a flow rate of $1.2 \mathrm{ml} / \mathrm{min}$ in an HPLC column over at $40^{\circ} \mathrm{C}$. The obtained chromatograms at $280 \mathrm{~nm}$ were used to obtain relative substrate conversion data, and additionally, control substrate and product samples were analyzed by ultraperformance liquid chromatography-MS to obtain ionization spectra.

\section{HPLC-based Michaelis-Menten assays}

Michaelis-Menten parameters for long peptide substrates were obtained by measuring conversion rates in a discontinuous manner. Assays were performed in Hepes buffer without BSA, where substrate (200 to $12.5 \mu \mathrm{M}$, twofold dilutions) and enzyme (HDAC1 with 6a: $150 \mathrm{nM}$; with 6b and 6c: $250 \mathrm{nM}$; HDAC2 with 6a: $150 \mathrm{nM}$; with 6b and 6c: $250 \mathrm{nM}$; or HDAC3 with 6a: $10 \mathrm{nM}$; with $\mathbf{6 b}, \mathbf{6 c}$, 10a, and 10c: $50 \mathrm{nM}$; and with 10b: $150 \mathrm{nM}$ ) were incubated in triplicate at $37^{\circ} \mathrm{C}$ in a final volume of $30 \mu \mathrm{l}$ in microcentrifuge tubes. Then, $\mathrm{MeOH} / \mathrm{HCOOH}[94: 6(\mathrm{v} / \mathrm{v})]$ was added to quench each of the reactions at 10-, 15-, and 20-min incubation times, respectively 
(15 $\mu \mathrm{l}$; for $45 \mu \mathrm{l}$ of total volume). Samples were then agitated on an orbital shaker, centrifuged at $20,000 \mathrm{~g}$ for $10 \mathrm{~min}$, taken up with a plastic syringe, filtered, and analyzed by analytical HPLC as before. The obtained chromatograms at $280 \mathrm{~nm}$ were used to obtain the relative substrate conversion at each time point. Then, if all three values followed a linear trend, then the obtained rates $\left(v_{0}\right)$ at each substrate concentration were adjusted to the Michaelis-Menten equation to afford $K_{\mathrm{M}}$ and $k_{\text {cat }}$ values. The entire experiment was performed at least twice.

\section{Histone extraction}

Histones were extracted using previously published method (54). Briefly, HeLa cells were harvested and washed twice with PBS

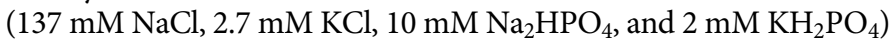
and then suspended in cold extraction buffer [10 mM Hepes ( $\mathrm{pH} 8.0$ ), $10 \mathrm{mM} \mathrm{KCl}, 1.5 \mathrm{mM} \mathrm{MgCl}_{2}, 0.34 \mathrm{M}$ sucrose, and $0.1 \%$ Triton X-100] at $4^{\circ} \mathrm{C}$ for $30 \mathrm{~min}$ to remove cytosolic components. After centrifugation at $1300 \mathrm{~g}$ for $10 \mathrm{~min}$ at $4^{\circ} \mathrm{C}$, the pellets were resuspended in no-salt buffer ( $3 \mathrm{mM}$ EDTA and $0.2 \mathrm{mM}$ EGTA) and incubated at $4^{\circ} \mathrm{C}$ for $30 \mathrm{~min}$ to release soluble nuclear components. The pellets were collected after centrifugation at $6500 \mathrm{~g}$ for $10 \mathrm{~min}$ at $4^{\circ} \mathrm{C}$ and then extracted with $0.2 \mathrm{M} \mathrm{H}_{2} \mathrm{SO}_{4}$ at $4^{\circ} \mathrm{C}$ overnight. The supernatants containing histones were collected after centrifugation at $16,000 \mathrm{~g}$ for $15 \mathrm{~min}$ at $4^{\circ} \mathrm{C}$. The resulting histones were further purified by precipitation using $20 \%(\mathrm{v} / \mathrm{v})$ trichloroacetic acid and washing with cold acetone and then dried and stored until further analysis.

\section{In vitro delactylation/acetylation assay using histones as substrates}

Histones extracted from HeLa cells $(20 \mu \mathrm{g})$ were incubated with individual recombinant human HDACs 1 to 11 or sirtuins 1 to 7 $(0.2 \mu \mathrm{g})$ in reaction buffer for 1 or 4 hours at $37^{\circ} \mathrm{C}$. HDACs 1 to 11 were tested using the standard tris buffer, and SIRTs 1 to 7 were tested in $50 \mathrm{mM}$ tris $\mathrm{HCl}$ ( $\mathrm{pH} 8.0$ ) with $1 \mathrm{mM}$ dithiothreitol (DTT) and $1 \mathrm{mM} \mathrm{NAD}^{+}$. The reaction was ended by adding sample loading buffer and boiling for $5 \mathrm{~min}$ before Western blot analysis.

\section{Peptide enrichment}

Histones extracted from HeLa cells were dissolved in $50 \mathrm{mM}$ $\mathrm{NH}_{4} \mathrm{HCO}_{3}$ buffer and digested with trypsin (Promega) as per the manufacturer's instructions. The digested histone peptides were incubated with pan anti-Kla beads at $4^{\circ} \mathrm{C}$ overnight. The beads were then washed twice with NETN buffer [ $50 \mathrm{mM}$ tris $\mathrm{HCl}$ ( $\mathrm{pH} 8.0$ ), $100 \mathrm{mM}$ $\mathrm{NaCl}, 1 \mathrm{mM}$ EDTA, and 0.5\% NP-40], once with ETN buffer [50 mM tris $\mathrm{HCl}(\mathrm{pH} 8.0), 100 \mathrm{mM} \mathrm{NaCl}$, and $1 \mathrm{mM}$ EDTA], and twice with $\mathrm{ddH}_{2} \mathrm{O}$. The peptides were eluted with $0.1 \%(\mathrm{v} / \mathrm{v}) \mathrm{TFA}$ and dried in a SpeedVac system (Thermo Fisher Scientific).

\section{HPLC-MS/MS analysis}

The resulting peptide samples were dissolved in buffer A $(0.1 \%$ formic acid in water, $\mathrm{v} / \mathrm{v}$ ) and loaded onto a homemade capillary column (10-cm length with 75- $\mu$ m ID) packed with Jupiter C12 resin (4- $\mu \mathrm{m}$ particle size and $90-\AA$ A pore size, Phenomenex) connected to an EASY-nLC 1000 HPLC system (Thermo Fisher Scientific). Peptides were separated and eluted with a gradient of 2 to $90 \%$ buffer $B$ $(0.1 \%$ formic acid in $90 \%$ acetonitrile, $\mathrm{v} / \mathrm{v})$ in buffer $\mathrm{A}(0.1 \%$ formic acid in water, v/v) at a flow rate of $200 \mathrm{nl} \mathrm{min}{ }^{-1}$ over $60 \mathrm{~min}$. The eluted peptides were then ionized and analyzed by a Q-Exactive mass spectrometer (Thermo Fisher Scientific). Full MS was acquired in the Orbitrap mass analyzer over the range $m / z$ (mass/charge ratio) 300 to 1400 with a resolution of 70,000 at $m / z 200$. The 12 most intense ions with charge $\geq 2$ were fragmented with normalized collision energy of 27 , and tandem mass spectra were acquired with a mass resolution of 17,500 at $m / z 200$. The raw data were uploaded into MaxQuant and searched against the UniProt human proteome database.

\section{Cell culture and transfection}

HeLa (ATCC CCL-2) cells were cultured in DMEM high-glucose medium (Gibco) containing 10\% FBS (GeminiBio), 1\% GlutaMAX (Gibco), and 5\% $\mathrm{CO}_{2}$. DNA and small interfering RNA (siRNA) transient transfection were performed using Lipofectamine 2000 (Invitrogen) according to the manufacturer's instructions. siRNAs targeting human HDAC1, HDAC2, and HDAC3 were purchased from GenePharma (Shanghai, China) as a SMART pool.

\section{Western blot analysis}

Protein samples ( $20 \mu \mathrm{g}$ of whole-cell lysate or $2 \mu \mathrm{g}$ of histone) were separated on SDS-polyacrylamide gel electrophoresis gels $(10 \%$ for nonhistones and $15 \%$ for histones) and then transferred to polyvinylidene fluoride membranes. The membranes were blocked in $3 \%$ BSA diluted in TBST [ $20 \mathrm{mM}$ tris/ $\mathrm{HCl}$ (pH 7.6), $150 \mathrm{mM} \mathrm{NaCl}$, and $0.1 \%$ Tween 20] for 1 hour at room temperature. The membranes were then incubated with primary antibody overnight at $4^{\circ} \mathrm{C}$. The blots were washed with TBST and then incubated for 1 hour with rocking in LI-COR secondary antibodies used at a concentration of 1:10,000. Western blots were developed using a LI-COR Odyssey.

\section{Immunofluorescence staining}

HeLa cells grown on coverslips were washed with PBS before fixation with $4 \%$ paraformaldehyde in PBS for $30 \mathrm{~min}$ at room temperature. After rinsing with PBS twice, the coverslips were incubated with $1 \%$ Triton X-100 for 15 min on ice, blocked with 5\% BSA for $60 \mathrm{~min}$ at $37^{\circ} \mathrm{C}$ incubator, and incubated with primary antibodies for 2 hours at room temperature. The coverslips were washed three times with PBST, followed by incubation with Texas Greenconjugated secondary antibodies. Images were acquired with an Olympus microscope system.

\section{Treatment with DD-I22 and SILAC-MS quantification}

HEK293 cells were cultured in either heavy (L-lysine- ${ }^{13} \mathrm{C}_{6},{ }^{15} \mathrm{~N}_{2}$, or K8) or light (L-lysine- ${ }^{12} \mathrm{C}_{6},{ }^{14} \mathrm{~N}_{2}$, or K0) DMEM, supplemented with $10 \%$ dialyzed FBS, for more than six passages to achieve more than 99\% labeling efficiency. Light-labeled cells were treated with PROTAC DD-I22 at $500 \mathrm{nM}$ concentration for 0,2 , or 4 hours, respectively. Heavy-labeled cells were treated with DMSO for 4 hours. Histones from both light- and heavy-labeled cells were acid extracted as described and mixed with 1:1 ratio. The mixed histones were then digested with trypsin, immunoprecipitated using a pan anti-Kla antibody, and analyzed by HPLC-tandem MS (MS/MS) as described. Quantification was analyzed by MaxQuant, and ratio H/L derived from MaxQuant was then normalized by protein abundance (median ratio was calculated from all peptides).

\section{Molecular docking}

Molecular modeling was performed with modules from the Schrödinger Small Molecule Drug Discovery Suite (Maestro), release 
2018-3, using the OPLS3 force field for parameterization. The $\mathrm{x}$-ray cocrystal structure of the HDAC3/SMRT-DAD complex (pdb 4A69) was imported from the pdb and prepared using the Protein Preparation Wizard using default settings. Ligands (2a, 2b, and 2c) were prepared using LigPrep. A receptor-grid box was built in a $25-\AA$ radius around $\mathrm{His}^{172}$ in the catalytic lysine-binding pocket, and docking was performed using the extra precision (XP) mode in Glide (74). Given the flexibility of a 3-mer peptide, numerous high-scoring binding conformations were predicted (XP score: $>9.5 \mathrm{kcal} / \mathrm{mol}$ ), so to assess the likely binding conformation, the binding modes were superpositioned with the backbone conformation of the residues of SMRT-DAD (derived from $x$-ray cocrystal structure) to assess the reasonability of the binding modes and select the most likely binding mode. Last, the ligand and residues of HDAC 3 in 5- $\AA$ proximity were refined and rescored using the MM-GBSA module in Prime (75). Figures were generated using PyMOL Molecular Graphics System (version 2.3.4, Schrödinger LLC).

\section{SUPPLEMENTARY MATERIALS}

Supplementary material for this article is available at https://science.org/doi/10.1126/ sciadv.abi6696

View/request a protocol for this paper from Bio-protocol.

\section{REFERENCES AND NOTES}

1. K. L. Diehl, T. W. Muir, Chromatin as a key consumer in the metabolite economy. Nat. Chem. Biol. 16, 620-629 (2020).

2. R. E. Moellering, B. F. Cravatt, Functional lysine modification by an intrinsically reactive primary glycolytic metabolite. Science 341, 549-553 (2013).

3. B. M. Dancy, P. A. Cole, Protein lysine acetylation by p300/CBP. Chem. Rev. 115 2419-2452 (2015).

4. M. Luo, Chemical and biochemical perspectives of protein lysine methylation. Chem. Rev. 118, 6656-6705 (2018).

5. C. Lu, C. B. Thompson, Metabolic regulation of epigenetics. Cell Metab. 16, 9-17 (2012).

6. J. C. Schell, D. R. Wisidagama, C. Bensard, H. Zhao, P. Wei, J. Tanner, A. Flores, J. Mohlman, L. K. Sorensen, C. S. Earl, K. A. Olson, R. Miao, T. C. Waller, D. Delker, P. Kanth, L. Jiang, R. J. DeBerardinis, M. P. Bronner, D. Y. Li, J. E. Cox, H. R. Christofk, W. E. Lowry, C. S. Thummel, J. Rutter, Control of intestinal stem cell function and proliferation by mitochondrial pyruvate metabolism. Nat. Cell Biol. 19, 1027-1036 (2017).

7. B. Faubert, K. Y. Li, L. Cai, C. T. Hensley, J. Kim, L. G. Zacharias, C. Yang, Q. N. Do, S. Doucette, D. Burguete, H. Li, G. Huet, Q. Yuan, T. Wigal, Y. Butt, M. Ni, J. Torrealba, D. Oliver, R. E. Lenkinski, C. R. Malloy, J. W. Wachsmann, J. D. Young, K. Kernstine, R. J. DeBerardinis, Lactate metabolism in human lung tumors. Cell 171, 358-371.e9 (2017).

8. J. D. Rabinowitz, S. Enerback, Lactate: The ugly duckling of energy metabolism. Nat. Metab. 2, 566-571 (2020).

9. O. Warburg, F. Wind, E. Negelein, The metabolism of tumors in the body. J. Gen. Physiol. 8, 519-530 (1927).

10. S. Hui, J. M. Ghergurovich, R. J. Morscher, C. Jang, X. Teng, W. Lu, L. A. Esparza, T. Reya, le Zhan, J. Yanxiang Guo, E. White, J. D. Rabinowitz, Glucose feeds the TCA cycle via circulating lactate. Nature 551, 115-118 (2017).

11. Z. Chen, M. Liu, L. Li, L. Chen, Involvement of the Warburg effect in non-tumor diseases processes. J. Cell. Physiol. 233, 2839-2849 (2018).

12. D. Zhang, Z. Tang, H. Huang, G. Zhou, C. Cui, Y. Weng, W. Liu, S. Kim, S. Lee, M. Perez-Neut, J. Ding, D. Czyz, R. Hu, Z. Ye, M. He, Y. G. Zheng, H. A. Shuman, L. Dai, B. Ren, R. G. Roeder, L. Becker, Y. Zhao, Metabolic regulation of gene expression by histone lactylation. Nature 574, 575-580 (2019).

13. E. L. Varner, S. Trefely, D. Bartee, E. von Krusenstiern, L. Izzo, C. Bekeova, R. S. O'Connor, E. L. Seifert, K. E. Wellen, J. L. Meier, N. W. Snyder, Quantification of lactoyl-CoA (lactyl-CoA) by liquid chromatography mass spectrometry in mammalian cells and tissues. Open Biol. 10, 200187 (2020).

14. B. R. Sabari, D. Zhang, C. D. Allis, Y. Zhao, Metabolic regulation of gene expression through histone acylations. Nat. Rev. Mol. Cell Biol. 18, 90-101 (2017).

15. S. Walenta, M. Wetterling, M. Lehrke, G. Schwickert, K. Sundfør, E. K. Rofstad, W. Mueller-Klieser, High lactate levels predict likelihood of metastases, tumor recurrence, and restricted patient survival in human cervical cancers. Cancer Res. 60, 916-921 (2000).

16. J. B. Ewaschuk, J. M. Naylor, G. A. Zello, D-lactate in human and ruminant metabolism. J. Nutr. 135, 1619-1625 (2005).
17. J. A. Landro, E. J. Brush, J. W. Kozarich, Isomerization of (R)- and (S)-glutathiolactaldehydes by glyoxalase I: The case for dichotomous stereochemical behavior in a single active site. Biochemistry 31, 6069-6077 (1992).

18. M. Sousa Silva, R. A. Gomes, A. E. Ferreira, A. Ponces Freire, C. Cordeiro, The glyoxalase pathway: The first hundred years... and beyond. Biochem. J. 453, 1-15 (2013).

19. G. R. Monroe, A. M. van Eerde, F. Tessadori, K. J. Duran, S. M. C. Savelberg, J. C. van Alfen, P. A. Terhal, S. N. van der Crabben, K. D. Lichtenbelt, S. A. Fuchs, J. Gerrits, M. J. van Roosmalen, K. L. van Gassen, M. van Aalderen, B. G. Koot, M. Oostendorp, M. Duran, G. Visser, T. J. de Koning, F. Calì, P. Bosco, K. Geleijns, M. G. M. de Sain-van der Velden, N. V. Knoers, J. Bakkers, N. M. Verhoeven-Duif, G. van Haaften, J. J. Jans, Identification of human D lactate dehydrogenase deficiency. Nat. Commun. 10, 1477 (2019).

20. D. O. Gaffney, E. Q. Jennings, C. C. Anderson, J. O. Marentette, T. Shi, A. M. Schou Oxvig M. D. Streeter, M. Johannsen, D. A. Spiegel, E. Chapman, J. R. Roede, J. J. Galligan, Non-enzymatic lysine lactoylation of glycolytic enzymes. Cell Chem. Biol. 27, 206-213.e6 (2020).

21. X. J. Yang, E. Seto, The Rpd3/Hda1 family of lysine deacetylases: From bacteria and yeast to mice and men. Nat. Rev. Mol. Cell Biol. 9, 206-218 (2008).

22. N. Rajabi, I. Galleano, A. S. Madsen, C. A. Olsen, Targeting sirtuins: Substrate specificity and inhibitor design. Prog. Mol. Biol. Transl. Sci. 154, 25-69 (2018).

23. J. Du, Y. Zhou, X. Su, J. J. Yu, S. Khan, H. Jiang, J. Kim, J. Woo, J. H. Kim, B. H. Choi, B. He, W. Chen, S. Zhang, R. A. Cerione, J. Auwerx, Q. Hao, H. Lin, Sirt5 is a NAD-dependent protein lysine demalonylase and desuccinylase. Science 334, 806-809 (2011).

24. C. Peng, Z. Lu, Z. Xie, Z. Cheng, Y. Chen, M. Tan, H. Luo, Y. Zhang, W. He, K. Yang, B. M. M. Zwaans, D. Tishkoff, L. Ho, D. Lombard, T.-C. He, J. Dai, E. Verdin, Y. Ye, Y. Zhao, The first identification of lysine malonylation substrates and its regulatory enzyme. Mol. Cell. Proteomics 10, M111.012658 (2011).

25. A. S. Madsen, C. A. Olsen, Substrates for efficient fluorometric screening employing the NAD-dependent sirtuin 5 lysine deacylase (KDAC) enzyme. J. Med. Chem. 55, 5582-5590 (2012).

26. M. Tan, C. Peng, K. A. Anderson, P. Chhoy, Z. Xie, L. Dai, J. Park, Y. Chen, H. Huang, Y. Zhang, J. Ro, G. R. Wagner, M. F. Green, A. S. Madsen, J. Schmiesing, B. S. Peterson, G. Xu, O. R. Ilkayeva, M. J. Muehlbauer, T. Braulke, C. Mühlhausen, D. S. Backos, C. A. Olsen, P. J. McGuire, S. D. Pletcher, D. B. Lombard, M. D. Hirschey, Y. Zhao, Lysine glutarylation is a protein posttranslational modification regulated by SIRT5. Cell Metab. 19, 605-617 (2014).

27. Y. B. Teng, H. Jing, P. Aramsangtienchai, B. He, S. Khan, J. Hu, H. Lin, Q. Hao, Efficient demyristoylase activity of SIRT2 revealed by kinetic and structural studies. Sci. Rep. $\mathbf{5}$, 8529 (2015).

28. P. Aramsangtienchai, N. A. Spiegelman, B. He, S. P. Miller, L. Dai, Y. Zhao, H. Lin, HDAC8 catalyzes the hydrolysis of long chain fatty acyl lysine. ACS Chem. Biol. 11, 2685-2692 (2016).

29. C. Moreno-Yruela, I. Galleano, A. S. Madsen, C. A. Olsen, Histone deacetylase 11 is an $\varepsilon-\mathrm{N}$-myristoyllysine hydrolase. Cell Chem. Biol. 25, 849-856.e8 (2018).

30. J. Cao, L. Sun, P. Aramsangtienchai, N. A. Spiegelman, X. Zhang, W. Huang, E. Seto, H. Lin, HDAC11 regulates type I interferon signaling through defatty-acylation of SHMT2. Proc. Natl. Acad. Sci. U.S.A. 116, 5487-5492 (2019).

31. A. S. Madsen, C. A. Olsen, Profiling of substrates for zinc-dependent lysine deacylase enzymes: HDAC3 exhibits decrotonylase activity in vitro. Angew. Chem. Int. Ed. 124 9217-9221 (2012).

32. W. Wei, X. Liu, J. Chen, S. Gao, L. Lu, H. Zhang, G. Ding, Z. Wang, Z. Chen, T. Shi, J. Li, J. Yu, J. Wong, Class I histone deacetylases are major histone decrotonylases: Evidence for critical and broad function of histone crotonylation in transcription. Cell Res. 27, 898-915 (2017).

33. R. Fellows, J. Denizot, C. Stellato, A. Cuomo, P. Jain, E. Stoyanova, S. Balázsi, Z. Hajnády, A. Liebert, J. Kazakevych, H. Blackburn, R. O. Corrêa, J. L. Fachi, F. T. Sato, W. R. Ribeiro, C. M. Ferreira, H. Perée, M. Spagnuolo, R. Mattiuz, C. Matolcsi, J. Guedes, J. Clark, M. Veldhoen, T. Bonaldi, M. A. R. Vinolo, P. Varga-Weisz, Microbiota derived short chain fatty acids promote histone crotonylation in the colon through histone deacetylases. Nat. Commun. 9, 105 (2018).

34. H. Huang, D. Zhang, Y. Weng, K. Delaney, Z. Tang, C. Yan, S. Qi, C. Peng, P. A. Cole, R. G. Roeder, Y. Zhao, The regulatory enzymes and protein substrates for the lysine beta-hydroxybutyrylation pathway. Sci. Adv. 7, eabe2771 (2021).

35. J. E. Bradner, N. West, M. L. Grachan, E. F. Greenberg, S. J. Haggarty, T. Warnow, R. Mazitschek, Chemical phylogenetics of histone deacetylases. Nat. Chem. Biol. 6, 238-243 (2010).

36. M. D. Jackson, M. T. Schmidt, N. J. Oppenheimer, J. M. Denu, Mechanism of nicotinamide inhibition and transglycosidation by Sir2 histone/protein deacetylases. J. Biol. Chem. 278, 50985-50998 (2003)

37. I. Galleano, M. Schiedel, M. Jung, A. S. Madsen, C. A. Olsen, A continuous, fluorogenic sirtuin 2 deacylase assay: Substrate screening and inhibitor evaluation. J. Med. Chem. 59, 1021-1031 (2016).

38. N. Rajabi, M. Auth, K. R. Troelsen, M. Pannek, D. P. Bhatt, M. Fontenas, M. D. Hirschey, C. Steegborn, A. S. Madsen, C. A. Olsen, Mechanism-based inhibitors of the human sirtuin 
5 deacylase: Structure-activity relationship, biostructural, and kinetic insight. Angew. Chem. Int. Ed. 56, 14836-14841 (2017).

39. I. Galleano, J. Nielsen, A. Madsen, C. Olsen, Scalable and purification-free synthesis of a myristoylated fluorogenic sirtuin substrate. Synlett 28, 2169-2173 (2017).

40. A. S. Madsen, C. Andersen, M. Daoud, K. A. Anderson, J. S. Laursen, S. Chakladar, F. K. Huynh, A. R. Colaço, D. S. Backos, P. Fristrup, M. D. Hirschey, C. A. Olsen, Investigating the sensitivity of NAD+-dependent sirtuin deacylation activities to NADH. J. Biol. Chem. 291, 7128-7141 (2016).

41. C. Moreno-Yruela, C. A. Olsen, High-throughput screening of histone deacetylases and determination of kinetic parameters using fluorogenic assays. STAR Protocols $\mathbf{2}$, 100313 (2021).

42. E. Q. Jennings, J. D. Ray, C. J. Zerio, M. N. Trujillo, D. M. McDonald, E. Chapman, D. A. Spiegel, J. J. Galligan, Sirtuin 2 regulates protein LactoylLys modifications. Chembiochem 22, 2102-2106 (2021).

43. X. Zhang, R. Cao, J. Niu, S. Yang, H. Ma, S. Zhao, H. Li, Molecular basis for hierarchical histone de- $\beta$-hydroxybutyrylation by SIRT3. Cell Discov. 5, 35 (2019).

44. M. G. Guenther, O. Barak, M. A. Lazar, The SMRT and N-CoR corepressors are activating cofactors for histone deacetylase 3. Mol. Cell. Biol. 21, 6091-6101 (2001).

45. B. R. Sabari, Z. Tang, H. Huang, V. Yong-Gonzalez, H. Molina, H. E. Kong, L. Dai, M. Shimada, J. R. Cross, Y. Zhao, R. G. Roeder, C. D. Allis, Intracellular crotonyl-CoA stimulates transcription through p300-catalyzed histone crotonylation. Mol. Cell 58, 203-215 (2015).

46. S. V. Olesen, N. Rajabi, B. Svensson, C. A. Olsen, A. S. Madsen, An NAD+-dependent sirtuin depropionylase and deacetylase (Sir2La) from the Probiotic bacterium lactobacillus acidophilusNCFM. Biochemistry 57, 3903-3915 (2018).

47. L. Dai, C. Peng, E. Montellier, Z. Lu, Y. Chen, H. Ishii, A. Debernardi, T. Buchou, S. Rousseaux, F. Jin, B. R. Sabari, Z. Deng, C. D. Allis, B. Ren, S. Khochbin, Y. Zhao, Lysine 2-hydroxyisobutyrylation is a widely distributed active histone mark. Nat. Chem. Biol. 10, 365-370 (2014).

48. Z. Xie, D. Zhang, D. Chung, Z. Tang, H. Huang, L. Dai, S. Qi, J. Li, G. Colak, Y. Chen, C. Xia, C. Peng, H. Ruan, M. Kirkey, D. Wang, L. M. Jensen, O. K. Kwon, S. Lee, S. D. Pletcher, M. Tan, D. B. Lombard, K. P. White, H. Zhao, J. Li, R. G. Roeder, X. Yang, Y. Zhao, Metabolic regulation of gene expression by histone lysine $\beta$-hydroxybutyrylation. Mol. Cell 62, 194-206 (2016).

49. J. L. Feldman, K. E. Dittenhafer-Reed, N. Kudo, J. N. Thelen, A. Ito, M. Yoshida, J. M. Denu, Kinetic and structural basis for acyl-group selectivity and $\mathrm{NAD}^{+}$dependence in sirtuincatalyzed deacylation. Biochemistry 54, 3037-3050 (2015)

50. C. A. Castañeda, N. A. Wolfson, K. R. Leng, Y.-M. Kuo, A. J. Andrews, C. A. Fierke, HDAC8 substrate selectivity is determined by long- and short-range interactions leading to enhanced reactivity for full-length histone substrates compared with peptides. J. Biol. Chem 292, 21568-21577 (2017).

51. P. J. Watson, L. Fairall, G. M. Santos, J. W. Schwabe, Structure of HDAC3 bound to co-repressor and inositol tetraphosphate. Nature 481, 335-340 (2012).

52. A. Vannini, C. Volpari, P. Gallinari, P. Jones, M. Mattu, A. Carfí, R. de Francesco, C. Steinkühler, S. di Marco, Substrate binding to histone deacetylases as shown by the crystal structure of the HDAC8-substrate complex. EMBO Rep. 8, 879-884 (2007).

53. S. M. L. Gantt, C. Decroos, M. S. Lee, L. E. Gullett, C. M. Bowman, D. W. Christianson, C. A. Fierke, General base-general acid catalysis in human histone deacetylase 8. Biochemistry 55, 820-832 (2016).

54. D. Shechter, H. L. Dormann, C. D. Allis, S. B. Hake, Extraction, purification and analysis of histones. Nat. Protoc. 2, 1445-1457 (2007)

55. S. E. Ong, B. Blagoev, I. Kratchmarova, D. B. Kristensen, H. Steen, A. Pandey, M. Mann, Stable isotope labeling by amino acids in cell culture, SILAC, as a simple and accurate approach to expression proteomics. Mol. Cell. Proteomics 1, 376-386 (2002).

56. C. Schölz, B. T. Weinert, S. A. Wagner, P. Beli, Y. Miyake, J. Qi, L. J. Jensen, W. Streicher, A. R. McCarthy, N. J. Westwood, S. Lain, J. Cox, P. Matthias, M. Mann, J. E. Bradner, C. Choudhary, Acetylation site specificities of lysine deacetylase inhibitors in human cells Nat. Biotechnol. 33, 415-423 (2015).

57. C. A. Hassig, J. K. Tong, T. C. Fleischer, T. Owa, P. G. Grable, D. E. Ayer, S. L. Schreiber, A role for histone deacetylase activity in HDAC1-mediated transcriptional repression. Proc. Natl. Acad. Sci. U.S.A. 95, 3519-3524 (1998)

58. C. C. Chini, C. Escande, V. Nin, E. N. Chini, HDAC3 is negatively regulated by the nuclear protein DBC1. J. Biol. Chem. 285, 40830-40837 (2010).

59. D. Martin, Y. Li, J. Yang, G. Wang, A. Margariti, Z. Jiang, H. Yu, A. Zampetaki, Y. Hu, Q. Xu L. Zeng, Unspliced X-box-binding protein 1 (XBP1) protects endothelial cells from oxidative stress through interaction with histone deacetylase 3. J. Biol. Chem. 289, 30625-30634 (2014).

60. C. J. Millard, P. J. Watson, L. Fairall, J. W. R. Schwabe, Targeting class I histone deacetylases in a "complex" environment. Trends Pharmacol. Sci. 38, 363-377 (2017).

61. Y. Xiao, J. Wang, L. Y. Zhao, X. Chen, G. Zheng, X. Zhang, D. Liao, Discovery of histone deacetylase 3 (HDAC3)-specific PROTACs. Chem. Commun. (Camb.) 56, 9866-9869 (2020).
62. M. Gao, J. Wang, S. Rousseaux, M. Tan, L. Pan, L. Peng, S. Wang, W. Xu, J. Ren, Y. Liu, M. Spinck, S. Barral, T. Wang, F. Chuffart, E. Bourova-Flin, D. Puthier, S. Curtet, L. Bargier, Z. Cheng, H. Neumann, J. Li, Y. Zhao, J. Q. Mi, S. Khochbin, Metabolically controlled histone H4K5 acylation/acetylation ratio drives BRD4 genomic distribution. Cell Rep. $\mathbf{3 6}$ 109460 (2021).

63. Z. A. Wang, C. J. Millard, C.-L. Lin, J. E. Gurnett, M. Wu, K. Lee, L. Fairall, J. W. Schwabe, P. A. Cole, Diverse nucleosome site-selectivity among histone deacetylase complexes. elife 9, e57663 (2020).

64. M. G. Riggs, R. G. Whittaker, J. R. Neumann, V. M. Ingram, n-Butyrate causes histone modification in HeLa and Friend erythroleukaemia cells. Nature 268, 462-464 (1977).

65. K. Steliou, M. S. Boosalis, S. P. Perrine, J. Sangerman, D. V. Faller, Butyrate histone deacetylase inhibitors. Biores Open Access 1, 192-198 (2012).

66. T. Latham, L. Mackay, D. Sproul, M. Karim, J. Culley, D. J. Harrison, L. Hayward, P. Langridge-Smith, N. Gilbert, B. H. Ramsahoye, Lactate, a product of glycolytic metabolism, inhibits histone deacetylase activity and promotes changes in gene expression. Nucleic Acids Res. 40, 4794-4803 (2012).

67. T. Shimazu, M. D. Hirschey, J. Newman, W. He, K. Shirakawa, N. le Moan, C. A. Grueter, H. Lim, L. R. Saunders, R. D. Stevens, C. B. Newgard, R. V. Farese Jr., R. de Cabo, S. Ulrich, K. Akassoglou, E. Verdin, Suppression of oxidative stress by $\beta$-hydroxybutyrate, an endogenous histone deacetylase inhibitor. Science 339, 211-214 (2013).

68. M. S. Verma, M. J. Fink, G. L. Salmon, N. Fornelos, T. E. Ohara, S. H. Ryu, H. Vlamakis, R. J. Xavier, T. S. Stappenbeck, G. M. Whitesides, A common mechanism links activities of butyrate in the colon. ACS Chem. Biol. 13, 1291-1298 (2018).

69. S. Chriett, A. Dąbek, M. Wojtala, H. Vidal, A. Balcerczyk, L. Pirola, Prominent action of butyrate over $\beta$-hydroxybutyrate as histone deacetylase inhibitor, transcriptional modulator and anti-inflammatory molecule. Sci. Rep. 9, 742 (2019).

70. M. Adeva-Andany, M. López-Ojén, R. Funcasta-Calderón, E. Ameneiros-Rodríguez, C. Donapetry-García, M. Vila-Altesor, J. Rodríguez-Seijas, Comprehensive review on lactate metabolism in human health. Mitochondrion 17, 76-100 (2014).

71. S. C. Kim, R. Sprung, Y. Chen, Y. Xu, H. Ball, J. Pei, T. Cheng, Y. Kho, H. Xiao, L. Xiao, N. V. Grishin, M. White, X. J. Yang, Y. Zhao, Substrate and functional diversity of lysine acetylation revealed by a proteomics survey. Mol. Cell 23, 607-618 (2006).

72. N. Rajabi, A. L. Nielsen, C. A. Olsen, Dethioacylation by sirtuins 1-3: Considerations for drug design using mechanism-based sirtuin inhibition. ACS Med. Chem. Lett. 11 1886-1892 (2020).

73. M. O. Lederer, Reactivity of lysine moieties toward $\gamma$-hydroxy- $\alpha, \beta$-unsaturated epoxides: A model study on protein-lipid oxidation product interaction. J. Agric. Food Chem. 44, 2531-2537 (1996).

74. W. Sherman, T. Day, M. P. Jacobson, R. A. Friesner, R. Farid, Novel procedure for modeling ligand/receptor induced fit effects. J. Med. Chem. 49, 534-553 (2006).

75. C. R. Guimaraes, M. Cardozo, MM-GB/SA rescoring of docking poses in structure-based lead optimization. J. Chem. Inf. Model. 48, 958-970 (2008).

Acknowledgments: We thank I. Galleano for donation of peptide substrates and S. A. Pless for donation of the HEK293T cell lines applied in the Olsen Lab, and we are grateful to P. Cole at Harvard for supply of the apicidin compound. Funding: This work was supported by the Ministry of Science and Technology of China (2017YFA054201; to J.W.), the Danish Council for Independent Research-Natural Sciences (grant no. 6108-00166B; to C.A.O.), the Independent Research Fund Denmark-Technical and Production Sciences (grant no. 0136-00412B; to C.A.O.), the Carlsberg Foundation (2013-01-0333 and CF15-011; to C.A.O.), the European Research Council (ERC-CoG-725172-SIRFUNCT; to C.A.O.), the University of Chicago, Nancy and Leonard Florsheim family fund (to Y.Z.), and the NIH (grants GM135504, AR078555, DK118266, and CA251677; to Y.Z.). Author contributions: Conceptualization: C.M.-Y., D.Z M.B., C.A.O., and Y.Z. Methodology: C.M.-Y., D.Z., W.W., M.B., J.G., D.D., A.L.N., C.A.O., and Y.Z. Formal analysis: C.M.-Y., D.Z., W.W., M.B., W.L., and J.G. Investigation: C.M.-Y., D.Z., W.W., M.B., W.L., J.G., D.D., A.L.N., J.E.B., and L.Y. Resources: C.M.-Y., D.Z., W.W., D.D., A.L.N., J.E.B., and S.T.J. Writing-original draft: C.M.-Y., D.Z., C.A.O., and Y.Z. Writing-review and editing: all authors. Visualization: C.M.-Y. and D.Z. Supervision: J.W., C.A.O., and Y.Z. Project administration: J.W., C.A.O., and Y.Z. Funding acquisition: J.W., C.A.O., and Y.Z. Competing interests: Y.Z. is a founder, board member, advisor to, and inventor on patents licensed to PTM Biolabs Inc. (Hangzhou, China and Chicago, IL) and Maponos Therapeutics Inc. (Chicago, IL). The other authors declare that they have no competing interests. Data and materials availability: All data needed to evaluate the conclusions in the paper are present in the paper and/or the Supplementary Materials.

Submitted 24 March 2021 Accepted 25 November 2021 Published 19 January 2022 10.1126/sciadv.abi6696 


\section{ScienceAdvances}

\section{Class I histone deacetylases (HDAC1-3) are histone lysine delactylases}

Carlos Moreno-YruelaDi ZhangWei WeiMichael BækWenchao LiuJinjun GaoDaniela DankováAlexander L. NielsenJulie E. BoldingLu YangSamuel T. JamesonJiemin WongChristian A. OlsenYingming Zhao

Sci. Adv., 8 (3), eabi6696. • DOI: 10.1126/sciadv.abi6696

\section{View the article online}

https://www.science.org/doi/10.1126/sciadv.abi6696

Permissions

https://www.science.org/help/reprints-and-permissions 